\title{
Quantum Continuum Mechanics Made Simple
}

\author{
Tim Gould, ${ }^{1}$ Georg Jansen, ${ }^{2}$ I. V. Tokatly, ${ }^{3,4}$ and John F. Dobson ${ }^{1}$ \\ ${ }^{1)}$ Qld Micro- and Nanotechnology Centre, Griffith University, Nathan, Qld 4111, \\ Australia \\ 2) Fakultät für Chemie, Universität Duisburg-Essen, 45117 Essen, Germany \\ ${ }^{3)}$ ETSF Scientific Development Centre, Departamento de Física de Materiales, \\ Universidad del País Vasco UPV/EHU, Av. Tolosa 72, E-20018 San Sebastián, \\ Spain \\ ${ }^{4)}$ IKERBASQUE, Basque Foundation for Science, E-48011, Bilbao, Spain
}

In this paper we further explore and develop the quantum continuum mechanics (QCM) of [Tao et al, PRL103,086401] with the aim of making it simpler to use in practice. Our simplifications relate to the non-interacting part of the QCM equations, and primarily refer to practical implementations in which the groundstate stress tensor is approximated by its Kohn-Sham version. We use the simplified approach to directly prove the exactness of QCM for one-electron systems via an orthonormal formulation. This proof sheds light on certain physical considerations contained in the QCM theory and their implication on QCM-based approximations. The one-electron proof then motivates an approximation to the QCM (exact under certain conditions) expanded on the wavefunctions of the Kohn-Sham (KS) equations. Particular attention is paid to the relationships between transitions from occupied to unoccupied KS orbitals and their approximations under the QCM. We also demonstrate the simplified QCM semi-analytically on an example system.

PACS numbers: 31.15.E-,31.15.ee,31.15.xg,31.15.ap

\section{INTRODUCTION}

The study of the continuum mechanics (fluid dynamics) of quantum electron fluids is almost as old as quantum mechnics itself. The Thomas-Fermi model and Madelung dynamics ${ }^{1}$ are two very early examples. Unfortunately standard approaches to Continuum Mechanics become too inaccurate or too complex when applied to larger, real systems such as molecules and atoms, although a recent development ${ }^{2}$ makes atomic systems more tractable. Recent work ${ }^{3-7}$ on Continuum Mechanics (QCM) in a moving Lagrangian frame has led to the development of a sophisticated approach for describing linear perturbations to many-electron systems from groundstate properties only. This approach appears able to bridge the gap between speed and accuracy required by modern $a b$ initio calculations.

The QCM provides an efficient ${ }^{7}$ alternative to full time-dependent density functional theory $(\operatorname{tdDFT})^{8-13}$ calculations. In its general form it can, in principle, be used to evaluate the transition frequencies and currents of a real many-electron quantum system with input from the interacting groundstate one-particle density matrix and two-particle density. In the formalism presented here we restrict to a more limited form that takes, as input, groundstate properties obtained from a Kohn-Sham ${ }^{8}$ calculation, and approximates small changes to the groundstate via a continuum approach. The ability to work with only groundstate KS properties as input comes at the expense of having to deal with higher order mixed derivatives (up to four derivatives with three indices). Initial indications suggest, however, that it is both tractable and valid in both model systems, ${ }^{5,6}$ as well as the difficult and geometrically very different case of two interacting twodimensional electron gas layers. ${ }^{7}$
Like Madelung dynamics, ${ }^{1}$ the recent QCM approach describes the behavior of the fluid displacement vector $\boldsymbol{u}$ from which the current and changes to the density can be described. As the independent-electron density response $\hat{\chi}_{0}$ of a system can be obtained from $\boldsymbol{u}$, a "QCMdRPA" correlation energy functional ${ }^{7}$ has been developed that uses the direct Random Phase Approximation (dRPA) but bypasses the need for unoccupied orbitals by working in the QCM directly via $\boldsymbol{u}$. The QCM-dRPA can be considered a "third-rung" functional according to the "Jacob's Ladder" classification scheme of Perdew et $a l .{ }^{14}$ The functional involves calculating the bare response via the QCM scheme and solving for the interacting response $\hat{\chi}_{\lambda}$ under the dRPA where additional interactions are treated at time-dependent Hartree level via $\hat{\chi}_{\lambda}=\hat{\chi}_{0}+\lambda \hat{\chi}_{0} \hat{v} \hat{\chi}_{\lambda}$. The response functions $\hat{\chi}_{0}$ and $\hat{\chi}_{\lambda}$ can then be used to calculate correlation energies. Here $\lambda$ is the strength of the interactions and must be integrated over (analytically in some formulations) to obtain the kinetic contribution to the correlation energy.

One particular area where it is hoped that the QCM will prove broadly useful is in the evaluation of van der Waals physics, where long-range correlation is important. Here local-density based techniques ${ }^{15-17}$ and even vdWadapted approximations like the vdW-DF ${ }^{18-21}$ run into difficulties (see Ref. 7 and Ref. 22 for discussion) because of their use of local or pairwise approximations.

The strong theoretical relationships between the QCM and the KS-like system it approximates, arising from its derivation from a formal, moving Lagrange frame, ${ }^{4}$ is somewhat hidden in the current prescription, especially to those used to working with orbitals and the Schrödinger equation. While relationships can be established (such as sum rules) between $\boldsymbol{u}$ and common groundstate properties of interest, they do not always 
come naturally from the original formulation.

Via adaptation and exploration of its theoretical form the QCM offers a wider scope for further investigation. This is undertaken in the present work, as follows:

Firstly, in their original form ${ }^{5}$ the QCM equations were complicated. In the first application to vdW physics, ${ }^{7}$ a more compact, simpler and more symmetric but equivalent form was used (equations 3-5 of Ref. 7). The derivation of this simplified form was not given in Ref. 7, and is presented here for the first time in Section II along with a discussion of the particular version of the groundstate stress tensor that makes this simplified version of the QCM theory possible. In Section III we discuss response functions and Adiabatic Connection/ Fluctuation Dissipation theory (ACFD) correlation energies, filling in some details not elaborated on in Ref. 7 .

Secondly, the standard displacement vector $\boldsymbol{u}$ has some undesirable properties in Coulomb-localised systems such as atoms and molecules, including divergence in the tail of the density distribution. To deal with this, in Section IV we reformulate the QCM in terms of an "orthonormal displacement vector" $\boldsymbol{\xi}=\sqrt{n^{0}} \boldsymbol{u}$ [touched on in equation (68) of Ref. 6] where $n^{0}$ is the electron density of the groundstate. This alternate approach opens the method up to expansion in basis sets with decaying tails, such as the widely used ${ }^{23}$ Gaussian-Type Orbitals (GTOs) and Slater-Type Orbitals (STOs) which are only valid in the expansion of decaying functions.

Thirdly, this reformulated QCM is used in Section V to demonstrate, directly from the Schrödinger equation, that the QCM gives the exact bare, linear response $\hat{\chi}_{0}$ for one-electron systems. While this relationship is established and demonstrated in earlier works (see equations (41)-(46) in Ref. 4, equation (98) and Appendix D in Ref. 5) a proof direct from the Schrödinger equation has not so far appeared. This relationship is very important, having as a consequence that properties dependent on $\hat{\chi}_{0}$ such as the (dRPA) asymptotic van der Waals interaction between atoms, are exactly reproduced by QCMdRPA theory for one-electron systems (and two-electron systems with equal groundstate densities of spin up and down electrons). The direct proof demonstrates how the QCM relates exactly to the first-order change of the oneelectron orbital/wavefunction.

Fourthly, the one-electron case is used in Section VI to motivate a second, approximate reformulation of the tensor QCM into a scalar system. Here the displacement $\boldsymbol{u}$ is approximated as the gradient of a scalar function $s$. This approach simplifies the QCM equations at the expense of accuracy in general systems but is exact for one-electron and one-dimensional systems. By then expanding $\sqrt{n^{0}} s$ on the set of KS orbitals we also uncover some of the physics of the QCM in the asymptotic tail regions of the density, where the electrons behave like a one-electron system.

Finally, in Section VII we illustrate the work on an example system: a one-dimensional, non-interacting Harmonic potential model. Here the one-electron case can be solved analytically while many of the terms in the many-electron case can be solved for analytically or using near exact quadrature, minimising numerical error. The scalar approximation is an exact reformulation of the QCM in this example.

\section{A. Notation}

In this paper we work entirely in atomic units where $m_{e^{-}}=\hbar=e^{2} /\left(4 \pi \epsilon_{0}\right)=1$ such that energies are in Hartee and distances in Bohr radii. We treat energies as frequencies with the division by $\hbar$ implicit.

Greek sub/super-scripts are used to refer to Cartesian $(x, y$ and $z$ ) coordinates and are summed over if repeated. We use the derivative operator notation $\left[\partial_{\mu} f(\boldsymbol{r})\right] \equiv\left[\frac{\partial f(\boldsymbol{r})}{\partial r_{\mu}}\right]$.

Cartesian tensors are written with sans serif letters (eg. $\mathrm{T}$ ), while Cartesian vectors appear in bold (eg. $\boldsymbol{v}$ ). Their elements are typically given as $T_{\mu \nu}$ and $v_{\alpha}$ respectively. The tensor $\mathrm{T}=\boldsymbol{u} \otimes \boldsymbol{v}$ has elements $T_{\mu \nu}=u_{\mu} v_{\nu}$. More general matrices use double-line Roman letters (eg. $\mathbb{M}$ ) and should be considered square unless otherwise noted.

Operators involve a hat eg. $\hat{O}$. If a derivative appears in an operator it can be considered to act entirely to the right unless surrounded by square brackets, but will act through other brackets. Thus $\left[\partial_{\alpha} v_{\alpha}\right] \equiv[\nabla \cdot \boldsymbol{v}]$, $\partial_{\mu} f \equiv\left[\partial_{\mu} f\right]+f \partial_{\mu}$ and $\left(\partial_{\mu} f\right) g \equiv\left[\partial_{\mu} f\right] g+f\left[\partial_{\mu} g\right]+f g \partial_{\mu}$. Comma-led subscripts will sometimes be used to represent derivatives $\left[\partial_{\mu} f_{\nu}\right] \equiv f_{\nu, \mu}$.

In the context of KS orbitals $j$ or $k$ can typically be any orbital but we reserve $i$ for occupied orbitals only and $a$ for unoccupied orbitals only such that $\sum_{i} \equiv \sum_{i \text { occ }}$ and $\sum_{a i} \equiv \sum_{i \text { occ }} \sum_{a \text { unocc }}$.

\section{ORIGINAL VS OPERATOR FORMS OF QCM}

The quantum continuum mechanics (QCM) formalism developed in previous works ${ }^{3-7}$ transforms the problem of calculating many-electron behaviour in a quantum mechanical system from an orbital approach to one in which the coordinate system itself is transformed via a displacement field $\boldsymbol{u}(\boldsymbol{r}, t) . \boldsymbol{u}$ was originally a classical concept, but it can nevertheless be defined rigorously for a time dependent many-body quantum state $|\Phi(t)\rangle$ as follows: ${ }^{24}$

$$
\begin{array}{lll}
\boldsymbol{u}(\boldsymbol{r}, t) & =\int_{t_{0}}^{t} \boldsymbol{v}\left(\boldsymbol{r}, t^{\prime}\right) \mathrm{d} t^{\prime}, & \boldsymbol{v}(\boldsymbol{r}, t)=\boldsymbol{j}(\boldsymbol{r}, t) / n(\boldsymbol{r}, t) \\
\boldsymbol{j}(\boldsymbol{r}, t)=\langle\Phi|\hat{\boldsymbol{j}}(\boldsymbol{r})| \Phi\rangle, & n(\boldsymbol{r}, t)=\langle\Phi|\hat{n}(\boldsymbol{r})| \Phi\rangle
\end{array}
$$

where $\hat{\boldsymbol{j}}(\boldsymbol{r})$ and $\hat{n}(\boldsymbol{r})$ are the standard current and density operators.

We can interpret $\boldsymbol{v}(\boldsymbol{r}, t)$ as the fluid velocity, and $\boldsymbol{u}(\boldsymbol{r}, t)$ as the displacement (from $\boldsymbol{r}$ ) at time $t$ of the fluid element that was at position $\boldsymbol{r}$ at time $t_{0}$. The continuity equation, ${ }^{24}$ along with (1), implies that, in a linear response situation around a stationary density $n^{0}(\boldsymbol{r})$, the 
current and density perturbation can be found from $\boldsymbol{u}$ through

$$
\begin{aligned}
\boldsymbol{j}(\boldsymbol{r}, t) & =\partial_{t} n^{0}(\boldsymbol{r}) \boldsymbol{u}(\boldsymbol{r}, t), \\
\partial_{t} n(\boldsymbol{r}, t) & =-\nabla \cdot \boldsymbol{j}(\boldsymbol{r}, t)
\end{aligned}
$$

and

$$
n^{1}(\boldsymbol{r}, t)=-\nabla \cdot n^{0}(\boldsymbol{r}) \boldsymbol{u}(\boldsymbol{r}, t) .
$$

The development of QCM started with work by Tokatly, ${ }^{3,4}$ who transformed the Schrödinger equation into the Lagrangian coordinate system that moves with a fluid element. The pressure tensor that determines the motion of $\boldsymbol{u}$ can be obtained in terms of derivatives of the energy in this frame with respect to $\boldsymbol{u}$, derivatives whose evaluation requires an analysis of the metric tensor $\mathrm{g}[\boldsymbol{u}]$ arising from the transformation from the rest frame to the Lagrangian frame. The QCM approximation ${ }^{5,6,25}$ takes the time dependent many-body wavefunction as a constant in the Lagrangian frame, corresponding to the fact that much of the motion of the rest-frame wavefunction is already dealt with via the motion of the fluid element. In the linear regime one can then explicitly evaluate the linear pressure tensor and force $\boldsymbol{F}^{1}$, without the metric tensor appearing explicitly. $\boldsymbol{F}^{1}$ involves as input only groundstate properties : density $n^{0}(\boldsymbol{r})$, Kohn-Sham potential $V^{\mathrm{KS}}(\boldsymbol{r})$, stress tensor $\mathrm{T}^{0}(\boldsymbol{r})$, and pair density $n_{2}^{0}\left(\boldsymbol{r}, \boldsymbol{r}^{\prime}\right)$. Here we are mainly interested in using $\boldsymbol{u}$ to calculate the bare (Kohn Sham) response, and for this purpose the pair distribution $n_{2}^{0}\left(\boldsymbol{r}, \boldsymbol{r}^{\prime}\right)$ is not needed.

Restricting to time-periodic perturbations of form $f(\boldsymbol{r}, t) \equiv f(\boldsymbol{r} ; \omega) e^{i \omega t}$, Gao et $a l^{6}$ showed that $\boldsymbol{u}(\boldsymbol{r} ; \omega)$ is governed by the elastic equation

$$
\omega^{2} n^{0} \boldsymbol{u}=\boldsymbol{F}^{1 \mathrm{Ext}}+\boldsymbol{F}^{1 \mathrm{Pot}}+\boldsymbol{F}^{1 \mathrm{Kin}}+\boldsymbol{F}^{1 \mathrm{Int}}
$$

which is exact for one-electron systems at all frequencies. For many-body systems it is exact in the limit of high frequency. Here the force terms are: the applied external force density $\boldsymbol{F}^{1 \mathrm{Ext}}(\boldsymbol{r} ; \omega)=n^{0}(\boldsymbol{r}) \nabla V^{1 \mathrm{Ext}}(\boldsymbol{r} ; \omega)$, the force from the distortion of the groundstate potential $\boldsymbol{F}^{1 \text { Pot }}(\boldsymbol{r} ; \omega)=n^{0}\left[\nabla \otimes \nabla V^{\mathrm{Ext}}(\boldsymbol{r})\right] \cdot \boldsymbol{u}(\boldsymbol{r} ; \omega)$, the force arising from changes to the kinetic energy $\boldsymbol{F}^{1 \mathrm{Kin}}(\boldsymbol{r} ; \omega)=$ $\delta \mathcal{T}_{2}[\boldsymbol{u}] / \delta \boldsymbol{u}(\boldsymbol{r} ; \omega)$, and the force arising from changes to the internal Coulomb interactions $\boldsymbol{F}^{1 \mathrm{Int}}(\boldsymbol{r} ; \omega)=$ $\delta \mathcal{W}_{2}[\boldsymbol{u}] / \delta \boldsymbol{u}(\boldsymbol{r} ; \omega) . \quad \mathcal{T}_{2}$ and $\mathcal{W}_{2}$ are both functionals of $\boldsymbol{u}(\boldsymbol{r} ; \omega)$.

The original papers ${ }^{5,6}$ establishing the linearised QCM theory give $\boldsymbol{F}^{1 \text { Kin }}$ in equation 14 of Ref. 5 (equation 53 of Ref. 6). Rearranging order a little this is

$$
\begin{aligned}
-F_{\mu}^{1 \text { Kin }}= & \partial_{\alpha}\left(2 \bar{T}_{\mu \nu}^{0} U_{\nu \alpha}+\bar{T}_{\nu \alpha}^{0} \partial_{\mu} u_{\nu}\right) \\
& +\frac{1}{4} \partial_{\nu}\left\{\left[\partial_{\nu} n^{0}\right] \partial_{\mu}+\left[\partial_{\mu} n^{0}\right] \partial_{\nu}-\partial_{\mu} n^{0} \partial_{\nu}\right\} \nabla \cdot \boldsymbol{u} \\
& +\frac{1}{2} \partial_{\nu}\left\{\left[\nabla^{2} n^{0}\right] U_{\mu \nu}-\partial_{\mu}\left(\left[\partial_{\alpha} n^{0}\right] U_{\nu \alpha}\right)\right\}
\end{aligned}
$$

Here $U_{\mu \nu}=\frac{1}{2}\left[\partial_{\nu} u_{\mu}+\partial_{\mu} u_{\nu}\right]$ and they define a 'kinetic stress tensor' $\bar{T}_{\mu \nu}$ [equation (17) in Ref. 6] via

$$
\begin{aligned}
\bar{T}_{\mu \nu}(\boldsymbol{r})= & \frac{1}{2}\left[\left(\partial_{\mu} \partial_{\nu}^{\prime}+\partial_{\mu}^{\prime} \partial_{\nu}\right) \rho\left(\boldsymbol{r}, \boldsymbol{r}^{\prime}\right)\right]_{\boldsymbol{r}=\boldsymbol{r}^{\prime}} \\
& -\frac{1}{4} \delta_{\mu \nu}\left[\nabla^{2} n(\boldsymbol{r})\right] .
\end{aligned}
$$

which is discussed in greater detail below. $\rho\left(\boldsymbol{r}, \boldsymbol{r}^{\prime}\right)$ is the one-particle density matrix of the system. $\overline{\mathrm{T}}^{0}$ in $(6)$ is the groundstate value of $\overline{\mathrm{T}}$.

Up to this point we have left the density, density matrix and potential terms undefined. In the formal theory these can be the exact quantities of the groundstate system, but in general these quantities are unknown. In a typical calculation it is likely that these would need to be calculated in a Kohn-Sham (KS) DFT based approximation, where we replace $V^{\text {Ext }}$ by the KS potential $V^{\mathrm{KS}}$ in the groundstate, and replace other quantities by their KS equivalents.

If we define a system with a KS potential $V^{\mathrm{KS}}$ (approximate or otherwise) then the one-electron Hamiltonian is

$$
\left\{-\frac{1}{2} \nabla^{2}+V^{\mathrm{KS}}(\boldsymbol{r})\right\} \psi_{j}(\boldsymbol{r})=\epsilon_{j} \psi_{j}(\boldsymbol{r})
$$

where $\psi_{j}(\boldsymbol{r})=\langle\boldsymbol{r} \mid j\rangle$ is a one-electron orbital which are orthonormal under $\int \mathrm{d} \boldsymbol{r} \psi_{j}^{*}(\boldsymbol{r}) \psi_{k}(\boldsymbol{r}) \equiv\langle j \mid k\rangle=\delta_{j k}$ and where $\epsilon_{j}$ is its Kohn-Sham eigenvalue.

The KS groundstate one-body density matric $\rho^{0}$ and one-body density $n^{0}$ are

$$
\begin{aligned}
\rho^{0}\left(\boldsymbol{r}, \boldsymbol{r}^{\prime}\right) & =\sum_{j} f_{j} \psi_{j}^{*}(\boldsymbol{r}) \psi_{j}\left(\boldsymbol{r}^{\prime}\right), \\
n^{0}(\boldsymbol{r}) & =\sum_{j} f_{j}\left|\psi_{j}(\boldsymbol{r})\right|^{2}=\rho^{0}(\boldsymbol{r}, \boldsymbol{r})
\end{aligned}
$$

where $f_{j}$ is the occupation of orbital $|j\rangle$ defined as 1 for orbitals with $\epsilon_{j}<\epsilon_{F}$ and 0 otherwise where $\epsilon_{F}$ is the Fermi energy. $\epsilon_{F}$ should be chosen to ensure that $\int n^{0}(\boldsymbol{r}) \mathrm{d} \boldsymbol{r}=N_{e}$ where $N_{e}$ is the total number of electrons.

\section{A. Kinetic stress tensor}

In general, the stress tensor is defined such that its divergence gives the force per unit volume. In a classical picture, the kinetic part $\mathrm{T}^{\mathrm{Kin}}$ of the stress tensor arises because each fluid element contains a spread of particle velocities, deviating from the mean value $\partial_{t} \boldsymbol{u}(\boldsymbol{r}, t)$ (the velocity of the fluid element). Because there are particles moving faster and slower than the fluid element, there is a leakage of particles into nearby fluid elements, and they bring their momentum with them, resulting in a force. Unsurprisingly, then, one way to obtain the elements of the classical stress tensor $T_{\mu \nu}^{\mathrm{Kin}}$ is to form a second momentum moment of the classical distribution 
function $f(\boldsymbol{r}, \boldsymbol{p}, t)$, multiplied by two factors of the momentum deviation vector, $\left(\boldsymbol{p}-m \partial_{t} \boldsymbol{u}\right)_{\mu}\left(\boldsymbol{p}-m \partial_{t} \boldsymbol{u}\right)_{\nu}-$ thus measuring a mean square spread of momenta. However there is still ambiguity because strictly only the divergence of $\mathrm{T}^{\mathrm{Kin}}$ is defined.

As a consequence of the above, any kinetic stress tensor $T^{\text {Kin }}$ must be real symmetric $\left[T_{\mu \nu}^{\mathrm{Kin}}(\boldsymbol{r})=T_{\nu \mu}^{\mathrm{Kin}}(\boldsymbol{r})\right]$ and obey the groundstate force balance condition $\left[\partial_{\alpha} \mathrm{T}_{\alpha \mu}^{\mathrm{Kin}}\right]=$ $-n^{0}\left[\partial_{\mu} V^{\mathrm{Ext}}\right]$.

For the kinetic stress tensor $\mathrm{T}$ corresponding to a oneelectron density matrix $\rho\left(\boldsymbol{r}, \boldsymbol{r}^{\prime}\right)$ in the absence of a current we choose the following definition, which we motivate and derive in Appendix A, consistently with the qualitative discussion above, and with the use of the Wigner distribution:

$$
T_{\mu \nu}^{\mathrm{Kin}}(\boldsymbol{r})=\left.\frac{1}{2}\left(\partial_{\mu} \partial_{\nu}^{\prime}+\partial_{\mu}^{\prime} \partial_{\nu}\right) \rho\left(\boldsymbol{r}, \boldsymbol{r}^{\prime}\right)\right|_{\boldsymbol{r}=\boldsymbol{r}^{\prime}}-\frac{1}{4} \partial_{\mu} \partial_{\nu} n(\boldsymbol{r}) .
$$

Inserting the density matrix from the independentelectron Kohn-Sham groundstate we obtain

$$
\begin{aligned}
T_{\mu \nu}^{0} & =\Re \sum_{j} f_{j}\left[\partial_{\mu} \psi_{j}^{*}\right]\left[\partial_{\nu} \psi_{j}\right]-\frac{1}{4} \partial_{\mu} \partial_{\nu} n^{0} \\
& \equiv \frac{1}{2} \Re \sum_{j} f_{j}\left(\left[\partial_{\mu} \psi_{j}^{*}\right]\left[\partial_{\nu} \psi_{j}\right]-\psi_{j}^{*}\left[\partial_{\mu} \partial_{\nu} \psi_{j}\right]\right)
\end{aligned}
$$

where terms are functions of $\boldsymbol{r}$ only.

This particular definition seems to allow for the most compact presentation of the QCM governing equations [e.g. (17) and (21), discussed later], and is favoured in this work for this reason. Unless otherwise noted we subsequently restrict ourselves to this form.

Earlier work on the QCM defines the kinetic stress tensor slightly differently [see (7)]. For an independentelectron Kohn-Sham groundstate this expression takes the form

$$
\bar{T}_{\mu \nu}^{0}=\Re \sum_{j} f_{j}\left[\partial_{\mu} \psi_{j}^{*}\right]\left[\partial_{\nu} \psi_{j}\right]-\frac{1}{4} \delta_{\mu \nu}\left[\nabla^{2} n^{0}\right]
$$

where terms depend on $\boldsymbol{r}$ only. The second components in (7) and (14) differ from ours [compare (11) and (12)].

\section{B. Linear QCM made simple}

Gould and Dobson ${ }^{7}$ noted without proof that $\boldsymbol{F}^{1 \text { Kin }} \equiv$ $-\hat{\mathrm{K}} \boldsymbol{u}$, where

$$
\hat{K}_{\mu \nu}=\partial_{\alpha} T_{\mu \nu}^{0} \partial_{\alpha}+\partial_{\alpha} T_{\alpha \nu}^{0} \partial_{\mu}+\partial_{\nu} T_{\alpha \mu}^{0} \partial_{\alpha}-\frac{1}{4} \partial_{\nu} \partial_{\alpha} n^{0} \partial_{\alpha} \partial_{\mu}
$$

is an Hermitian operator. A full derivation of this expression appears in Appendix B. Since $\boldsymbol{F}^{1 \mathrm{Pot}}=n^{0}[\nabla \otimes$ $\left.\nabla V^{\mathrm{KS}}\right] \cdot \boldsymbol{u}$, we can write $\boldsymbol{F}^{1 \mathrm{Pot}}+\boldsymbol{F}^{1 \mathrm{Kin}}=\hat{\mathrm{R}} \boldsymbol{u}$ and thus

$$
\begin{aligned}
\left(\omega^{2} n^{0}-\hat{\mathrm{R}}\right) \boldsymbol{u} & =F^{1 \mathrm{Ext}}+F^{1 \mathrm{Int}}, \\
\hat{R}_{\mu \nu} & =n^{0} V_{, \mu \nu}^{\mathrm{KS}}-\hat{K}_{\mu \nu}
\end{aligned}
$$

where all terms but $\omega$ vary with $\boldsymbol{r}$. The operator $\hat{R}$ is manifestly Hermitian, and can be shown to be positive definite. This form of the QCM is easier to deal with in numerical calculations, and lends itself nicely to expansion on an auxilliary basis set. ${ }^{7}$

Equation (15) leads to the same force as (6) but in a much simplified manner. Much of this simplification comes from the different choice of kinetic stress tensor, given by (11) and (12) in our work and by (7) and (14) in Refs 5 and 6, as discussed in Appendix B.

Equations 12, 15, 16 and 17, form the foundation of the remaining work in this manuscript. They form the first stage of the 'simplification' of the QCM.

\section{RESPONSE FUNCTIONS}

As mentioned in the introduction, one potential application of the QCM is in the evaluation of response functions, and through them the groundstate energy of many-electron systems. This approach was previously investigated $^{7}$ by some of the authors and found to work well in the two-dimensional jellium systems studied. Similarly certain exact properties of the response were previously investigated in Refs 5 and 6 . Here we spend some time expanding on this previous work.

Let us first look at response within the QCM, and then we will investigate how the QCM response relates to the true KS response. In (5) $\boldsymbol{F}^{1 \text { Int }}$ takes into account the electron-electron interaction, while $\hat{R} \boldsymbol{u}$ deals with kinetic and potential physics. The dRPA is equivalent to setting

$$
\begin{aligned}
\boldsymbol{F}^{1 \mathrm{Int}}(\boldsymbol{r}) & =[\hat{\mathrm{Q}} \boldsymbol{u}](\boldsymbol{r}) \\
& \equiv-n^{0}(\boldsymbol{r}) \nabla \int \frac{\mathrm{d} \boldsymbol{r}^{\prime}}{\left|\boldsymbol{r}-\boldsymbol{r}^{\prime}\right|}\left[\nabla^{\prime} \cdot n^{0}\left(\boldsymbol{r}^{\prime}\right) \boldsymbol{u}\left(\boldsymbol{r}^{\prime}\right)\right] \\
& \equiv \int \mathrm{d} \boldsymbol{r}^{\prime} \mathrm{Q}\left(\boldsymbol{r}, \boldsymbol{r}^{\prime}\right) \cdot \boldsymbol{u}\left(\boldsymbol{r}^{\prime}\right)
\end{aligned}
$$

where

$$
\mathrm{Q}\left(\boldsymbol{r}, \boldsymbol{r}^{\prime}\right)=n^{0}(\boldsymbol{r}) n^{0}\left(\boldsymbol{r}^{\prime}\right)\left[\nabla \otimes \nabla^{\prime} \frac{1}{\left|\boldsymbol{r}-\boldsymbol{r}^{\prime}\right|}\right] .
$$

In the absence of an external field $\boldsymbol{F}^{1 \mathrm{Ext}}=\mathbf{0}$ we can find eigen-mode pairs $\Omega_{N}$ and $\boldsymbol{u}_{N}$ (or $\Omega_{N \lambda}$ and $\boldsymbol{u}_{N \lambda}$ ) through solutions of

$$
\begin{aligned}
\Omega_{N}^{2} n^{0} \boldsymbol{u}_{N} & =\hat{\mathrm{R}} \boldsymbol{u}_{N}, \\
\Omega_{N \lambda}^{2} n^{0} \boldsymbol{u}_{N \lambda} & =\hat{\mathrm{R}} \boldsymbol{u}_{N \lambda}+\lambda \hat{\mathrm{Q}} \boldsymbol{u}_{N \lambda}
\end{aligned}
$$

where (22) includes the internal interactions $\lambda \hat{Q}$ at coupling strength $\lambda$ while we use the short-hand $\Omega_{N}=\Omega_{N 0}$ 
and $\boldsymbol{u}_{N}=\boldsymbol{u}_{N 0}$ for the non-interacting case. Because $\hat{\mathrm{R}}$ and $\hat{\mathrm{Q}}$ are Hermitian (and in fact can be shown ${ }^{6}$ to be positive definite) and $n^{0}$ is symmetric and positive definite, eigensolutions can be found that obey the orthonormality condition

$$
\int \mathrm{d} \boldsymbol{r} n^{0} \boldsymbol{u}_{N \lambda}^{*} \cdot \boldsymbol{u}_{M \lambda}=\delta_{N M}
$$

The set $\left\{\boldsymbol{u}_{N \lambda}\right\}$ is also guaranteed to be complete over a finite basis when $\hat{R}+\lambda \hat{Q}$ is represented in the same finite basis. Furthermore the eigen-values $\Omega_{N \lambda}^{2}$ must be positive. Typically we also sort the modes such that $\Omega_{N+1 \lambda} \geq \Omega_{N \lambda}$ where $N \geq 1$ labels the QCM mode with the $N$ th lowest energy. The displacement $\boldsymbol{u}_{N \lambda}$ corresponds to a transition density mode (the meaning will become clearer later) defined as

$$
d_{N \lambda}(\boldsymbol{r})=-\nabla \cdot n^{0}(\boldsymbol{r}) \boldsymbol{u}_{N \lambda}(\boldsymbol{r})
$$

If the external force density $\boldsymbol{F}^{1 \mathrm{Ext}}$ is reintroduced we can expand the solution of (5) at interaction strength $\lambda$ on the basis $\left\{\boldsymbol{u}_{N \lambda}\right\}$ such that $\boldsymbol{u}=\sum_{N} c_{N \lambda} \boldsymbol{u}_{N \lambda}$. Here

$$
c_{N \lambda}=\frac{\int \mathrm{d} \boldsymbol{r} n^{0}(\boldsymbol{r}) \boldsymbol{u}_{N \lambda}^{*}(\boldsymbol{r}) \cdot \boldsymbol{F}^{1 \mathrm{Ext}}(\boldsymbol{r})}{\Omega_{N \lambda}^{2}-\omega^{2}}
$$

when $\boldsymbol{F}^{1 \text { Ext }}$ is time-periodic with frequency $\omega$. The change in density (4) can thus be expanded on (24) as

$$
n_{\lambda}^{1}(\boldsymbol{r}, \omega)=\sum_{N} c_{N \lambda} d_{N \lambda}(\boldsymbol{r})
$$

where the sum is over all eigen-solutions.

The density response $\chi_{\lambda}$ of a system is defined as the change in density in response to a $\delta\left(\boldsymbol{r}-\boldsymbol{r}^{\prime}\right)$ potential at a frequency $\omega$ with internal interactions at strength $\lambda$. This corresponds to an external force $\boldsymbol{F}^{1 \mathrm{Ext}}=n^{0} \nabla \delta(\boldsymbol{r}-$ $\left.\boldsymbol{r}^{\prime}\right) e^{-i \omega t}$ and internal force $\boldsymbol{F}^{1 \mathrm{Int}}=\lambda \hat{\mathrm{Q}} \boldsymbol{u}(\omega) e^{-i \omega t}$. Thus the response takes the form $\hat{\chi}_{\lambda}(t)=\hat{\chi}_{\lambda}(\omega) e^{-i \omega t}$ where $\chi_{\lambda}\left(\boldsymbol{r}, \boldsymbol{r}^{\prime} ; \omega\right)=-\sum_{N} \frac{d_{N \lambda}^{*}(\boldsymbol{r}) d_{N \lambda}\left(\boldsymbol{r}^{\prime}\right)}{\Omega_{N \lambda}^{2}-\omega^{2}}$. Typically it is easier to work with responses at imaginary frequency $\omega=i \sigma$ such that

$$
\begin{aligned}
& \chi_{0}\left(\boldsymbol{r}, \boldsymbol{r}^{\prime} ; i \sigma\right)=-\Re \sum_{N} \frac{d_{N}^{*}(\boldsymbol{r}) d_{N}\left(\boldsymbol{r}^{\prime}\right)}{\sigma^{2}+\Omega_{N}^{2}}, \\
& \chi_{\lambda}\left(\boldsymbol{r}, \boldsymbol{r}^{\prime} ; i \sigma\right)=-\Re \sum_{N} \frac{d_{N \lambda}^{*}(\boldsymbol{r}) d_{N \lambda}\left(\boldsymbol{r}^{\prime}\right)}{\sigma^{2}+\Omega_{N \lambda}^{2}},
\end{aligned}
$$

where (27) uses the solutions of (21) to calculate the bare $(\lambda=0)$ response while (28) uses $(22)$ to solve directly for the interacting response. The $\Re$ is unnecessary as the sum itself can be guaranteed real but may prove useful in some situations.

While $\hat{\chi}_{\lambda}$ defined by (28) has useful formal properties its direct evaluation may be numerically difficult and can be avoided. Unless otherwise noted we henceforth set $\boldsymbol{F}^{1 \mathrm{Int}}=\mathbf{0}$ and deal with internal interactions (when required) in a less direct, but precisely equivalent and more computationally convenient manner (as discussed tangentially in Ref. 7 and in Appendix C of this manuscript).

\section{A. Relationships to $\mathrm{KS}$ response}

In a Kohn-Sham system with orbitals $\psi_{j}(\boldsymbol{r}) \equiv\langle\boldsymbol{r} \mid j\rangle$, the exact bare response takes the form ${ }^{9}$

$$
\chi_{0}^{\mathrm{KS}}\left(\boldsymbol{r}, \boldsymbol{r}^{\prime} ; i \sigma\right)=-\Re \sum_{a i} \frac{d_{a i}^{*}(\boldsymbol{r}) d_{a i}\left(\boldsymbol{r}^{\prime}\right)}{\sigma^{2}+\Omega_{a i}^{2}}
$$

where $d_{a i}$ is a normalised transition density between unoccupied orbital $|a\rangle$ and occupied orbital $|i\rangle$ while $\Omega_{a i}$ is the transition frequency defined by

$$
\begin{aligned}
d_{a i}(\boldsymbol{r}) & =\sqrt{2 \Omega_{a i}} \psi_{a}^{*}(\boldsymbol{r}) \psi_{i}(\boldsymbol{r}), \\
\Omega_{a i} & =\epsilon_{a}-\epsilon_{i}>0 .
\end{aligned}
$$

As noted in Sec. I A, $i$ is summed over occupied orbitals only and $a$ over unoccupied orbitals only.

There is a transition current density associated with $|a\rangle$ and $|i\rangle$ which takes the form

$$
\boldsymbol{j}_{a i}(\boldsymbol{r})=\frac{1}{2 i}\left[\psi_{a}(\boldsymbol{r}) \nabla \psi_{i}^{*}(\boldsymbol{r})-\psi_{i}^{*}(\boldsymbol{r}) \nabla \psi_{a}(\boldsymbol{r})\right]
$$

and where $i \nabla \cdot \boldsymbol{j}_{a i}=\Omega_{a i} \psi_{i} \psi_{a}^{*}=\sqrt{\Omega_{a i} / 2} d_{a i}$. Since $\left\{\boldsymbol{u}_{N}\right\}$ is complete (at least within a given finite basis) and orthonormal under (23) we can expand $\boldsymbol{j}_{a i}=$ $n^{0} \sum_{N}\left[\int \mathrm{d} \boldsymbol{r} \boldsymbol{u}_{N}^{*} \cdot \boldsymbol{j}_{a i}\right] \boldsymbol{u}_{N}$. Taking the gradient of $\boldsymbol{j}_{a i}$ thus provides the following relationship between the KS transition densities $d_{a i}$ and the QCM density modes $d_{N}$

$$
d_{a i}=\sum_{N} K_{a i N} d_{N}, \quad K_{a i N}=\frac{i \int \mathrm{d} \boldsymbol{r} \boldsymbol{u}_{N}^{*} \cdot \boldsymbol{j}_{a i}}{\sqrt{\Omega_{a i} / 2}}
$$

so that any $d_{a i}$ can be expanded in $\left\{d_{N}\right\}$. Unfortunately, since $\left\{d_{a i}\right\}$ is not necessarily complete, the converse cannot be guaranteed except in the trivial one-electron case.

Certain exact sum rules [equations (81)-(83) further discussed in appendix E of Ref. 6] provide some further restrictions on the various coefficients. Since the fand third-moment sum rules are satisfied by the QCM it follows ${ }^{5}$ that

$$
1=\sum_{a i}\left|K_{a i N}\right|^{2}, \quad \Omega_{N}^{2}=\sum_{a i}\left|K_{a i N}\right|^{2} \Omega_{a i}^{2}
$$

where $\Omega_{a i}$ are the Kohn-Sham transition frequencies of the system. For $N \neq M$ we find

$$
0=\sum_{a i} K_{a i N}^{*} K_{a i M}=\sum_{a i} K_{a i N}^{*} K_{a i M} \Omega_{a i}^{2}
$$

which come from inserting (32) into (29) and comparing the leading two powers of $1 / \sigma^{2}$ with $(27)$. 
The second sum rule in (33) makes the relationship between $\Omega_{N}$ and the KS transition frequencies clear. We may also consider $d_{N}$ to be an approximation to collections of the transition densities, with errors hopefully minimised by the sum rules and exact properties even though no direct expansion exists. As discussed later these approximations become exact for one-electron (or two-electrons with equal spin densities $n_{\uparrow}=n_{\downarrow}$ ) systems.

It is also worth noting that the lowest QCM transition frequency $\Omega_{1}$ can never be less than the transition frequency between the highest occupied- and lowest unuccopied- molecular orbital $\Omega_{L H}=\epsilon_{L}-\epsilon_{H}$. In the non-degenerate case the equality follows if and only if $\left|K_{L H 1}\right|^{2}=1$ with all other $K_{a i 1}$ zero. To prove the inequality we note that $\Omega_{a i} \geq \Omega_{L H}$ and thus

$$
\Omega_{1}^{2} \geq \sum_{a i}\left|K_{a i 1}\right|^{2} \Omega_{L H}^{2} \geq \Omega_{L H}^{2} .
$$

$$
\begin{aligned}
& \text { If }\left|K_{L H 1}\right|^{2}<1 \text { then }\left|K_{L H 1}\right|^{2}=1-\sum_{a i \neq L H}\left|K_{a i 1}\right|^{2} \text { and } \\
& \begin{aligned}
\Omega_{1}^{2}-\Omega_{L H}^{2} & =\left(\left|K_{L H 1}\right|^{2}-1\right) \Omega_{L H}^{2}+\sum_{a i \neq L H}\left|K_{a i 1}\right|^{2} \Omega_{a i}^{2} \\
& =\sum_{a i \neq L H}\left|K_{a i 1}\right|^{2}\left(\Omega_{a i}^{2}-\Omega_{L H}^{2}\right)>0
\end{aligned}
\end{aligned}
$$

since $\left|K_{a i 1}\right|^{2}>0$ and $\Omega_{a i}^{2}-\Omega_{L H}^{2}>0$. Thus the equality only holds if $\left|K_{a i 1}\right|^{2}=1$. A direct consequence of this is that a KS insulator will remain an insulator under the QCM.

\section{B. Correlation energies made simple}

From the bare and interacting response functions it is relatively straightforward to obtain exchange and correlation energies. In a true KS response formalism this can be obtained via the occupied and unoccupied orbitals. In the QCM these are replaced by $d_{N}$ and $\Omega_{N}$ and once these have been obtained the QCM approximation to the correlation energy can be calculated. ${ }^{7}$

We define the Coulomb projection matrix $\mathbb{W}$ with elements

$$
\begin{aligned}
W_{N M} & =\int \mathrm{d} \boldsymbol{r} \boldsymbol{u}_{M}^{*}(\boldsymbol{r}) \cdot\left[\hat{\mathbf{Q}} \boldsymbol{u}_{N}\right](\boldsymbol{r}) \\
& =\int \mathrm{d} \boldsymbol{r} \mathrm{d} \boldsymbol{r}^{\prime} \frac{d_{N}(\boldsymbol{r}) d_{M}^{*}\left(\boldsymbol{r}^{\prime}\right)}{\left|\boldsymbol{r}-\boldsymbol{r}^{\prime}\right|}
\end{aligned}
$$

and $\mathbb{L}$ with elements $L_{M N}=\delta_{M N} \Omega_{N}^{2}$. Through the working in Appendix $\mathrm{C}$ we can show that the correlation energy is

$$
\begin{aligned}
E_{\mathrm{c}}= & \int_{0}^{1} \mathrm{~d} \lambda \int_{0}^{\infty} \frac{\mathrm{d} \sigma}{\pi} \\
& \times \frac{1}{2} \operatorname{Tr}\left[\frac{\mathbb{W}}{\sigma^{2}+\mathbb{L}+\lambda \mathbb{W}}-\frac{\mathbb{W}}{\sigma^{2}+\mathbb{L}}\right]
\end{aligned}
$$

or we can use the Furche-like ${ }^{26}$ integrated form

$$
E_{\mathrm{c}}=\frac{1}{2} \sum_{N}\left[\bar{\Omega}_{N}-\Omega_{N}\left(1+\frac{W_{N N}}{2 \Omega_{N}^{2}}\right)\right]
$$

where $\bar{\Omega}_{N}^{2}$ are the eigenvalues of $\hat{\mathrm{R}}+\hat{\mathrm{Q}}$ or $\mathbb{L}+\mathbb{W}$. As discussed in Appendix $\mathrm{C}$ the two diagonalisations are formally equivalent but experience in similar techniques suggests that working in the transition densities of the bare response will allow for better convergence. In practice diagonalising $\mathbb{L}+\mathbb{W}$ is expected to be faster and numerically more reliable and robust.

Using the eigenvalues of $\mathbb{L}+\mathbb{W}$ has a further advantage: we can use a perturbative solution to find the eigenvalues of $\mathbb{L}+\mathbb{W}$ if $\Omega_{N}^{2} \gg \bar{W}_{N}$ where $\bar{W}_{N}=\sum_{M}\left|W_{N M}\right|$ (see Appendix $\mathrm{C}$ for details). We define an $N^{*}$ such that $\Omega_{N}^{2} \geq K \bar{W}_{N} \forall N>N^{*}$ where $K$ is sufficiently large. We then solve the reduced $N^{*} \times N^{*}$ eigen-equation $\mathbb{L}^{*}+\mathbb{W}^{*}$ to obtain $\bar{\Omega}_{N=1 \ldots N^{*}}^{*}$ and calculate

$$
E_{\mathrm{c}} \approx \frac{1}{2} \sum_{N=1}^{N^{*}}\left[\bar{\Omega}_{N}^{*}-\Omega_{N}\left(1+\beta_{N}\right)\right]-\sum_{N>N^{*}} \frac{\Omega_{N} \beta_{N}^{2}}{4}
$$

where $\beta_{N}=W_{N N} /\left(2 \Omega_{N}^{2}\right)$.

From the perspective of energy calculations, (39) is the second main stage of simplification of the QCM for practical purposes. Energies can be calculated through solutions of the QCM eigen-equation (21) using the simplified operator (15) in (17).

\section{ORTHONORMAL DISPLACEMENT}

So far we have investigated, and simplified for practical purposes, the QCM in its original context as a set of governing equations for the displacement $\boldsymbol{u}$ [see equations 12 , 15, 17 and 21]. From these we have derived other quantities of interest such as response functions and correlation energies. In this section we provide a reformulation of these equations designed to make applications to bound systems like atoms and molecules more tractable in general.

If we consider the orthonormality condition $\int \mathrm{d} \boldsymbol{r} n^{0} \boldsymbol{u}_{N}^{*}$. $\boldsymbol{u}_{M}=\delta_{N M}$ [Equation 23] on the displacement eigenmodes in a bound system, we can see that $\boldsymbol{u}_{N}$ may be permitted to grow as $|\boldsymbol{r}| \rightarrow \infty$ provided $\sqrt{n^{0}} \boldsymbol{u}_{N}$ decreases. In atomic and molecular systems all valid solutions will, in fact, grow exponentially due to the asymptotic form of the orbitals. While formally this is not a great concern, in practise it makes accurate calculation more difficult in finite systems.

The orthogonality condition suggests that we can define an orthonormal fluid displacement $\boldsymbol{\xi}=\sqrt{n^{0}} \boldsymbol{u}$ that will be a more natural quantity to use in these systems as it is guaranteed to decrease. Here the orthonormal eigen-modes are

$$
\boldsymbol{\xi}_{N}=\sqrt{n^{0}} \boldsymbol{u}_{N}
$$


where $\int \mathrm{d} \boldsymbol{r} \boldsymbol{\xi}_{N}^{*} \cdot \boldsymbol{\xi}_{M}=\delta_{N M}$

While $\boldsymbol{u}=\sum_{N} c_{N} \boldsymbol{u}_{N}$ has a well-defined physical meaning (the fluid displacement), $\boldsymbol{\xi}=\sum c_{N} \boldsymbol{\xi}_{N}$ is somewhat harder to interpret. However some insight can be gained if we define the groundstate quasi-orbital $\Psi^{0}=$ $\sqrt{n^{0}}$ and its first order change $\Psi^{1}$. Since $n^{1}=2 \Psi^{0} \Psi^{1}=$ $-\nabla \cdot \Psi^{0} \boldsymbol{\xi}$ it is clear that

$$
2 \Psi^{1}=-\left(\nabla+\frac{\left[\nabla \Psi^{0}\right]}{\Psi^{0}}\right) \cdot \boldsymbol{\xi}
$$

and thus $\boldsymbol{\xi}$ is related to the perturbation of the quasiorbital. It is also related, via (3), to the groundstate properties $n^{1}$ and $\boldsymbol{j}$ through

$$
\boldsymbol{j}=\partial_{t} \Psi^{0} \boldsymbol{\xi} \quad n^{1}=-\nabla \Psi^{0} \boldsymbol{\xi} .
$$

We must calculate $\boldsymbol{\xi}_{N}$ through the QCM equations (21) which can be rewritten as

$$
\begin{aligned}
\Omega_{N}^{2} \boldsymbol{\xi}_{N} & =\hat{\mathrm{R}}^{(\boldsymbol{\xi})} \boldsymbol{\xi}_{N}, \\
\hat{\mathrm{R}}^{(\boldsymbol{\xi})} & =\frac{1}{\Psi^{0}} \hat{\mathrm{R}} \frac{1}{\Psi^{0}}
\end{aligned}
$$

where $\hat{R}$ is defined in equation (17). The change in density of a given mode becomes

$$
d_{N}(\boldsymbol{r})=-\Psi^{0}(\nabla+\boldsymbol{\eta}) \cdot \boldsymbol{\xi}_{N}
$$

where

$$
\boldsymbol{\eta}=\frac{\left[\nabla n^{0}\right]}{2 n^{0}}=\frac{\left[\nabla \Psi^{0}\right]}{\Psi^{0}}=\nabla \log \Psi^{0}=\frac{1}{2} \nabla \log n^{0}
$$

is the logarithmic gradient of $\Psi^{0}$. Equation 46 can be used in (37) to calculate the matrix elements of $\mathbb{W}$ for use in correlation energy calculations

Inserting (17) into (45) gives

$$
\begin{aligned}
\hat{R}_{\mu \nu}^{(\boldsymbol{\xi})}= & -\frac{1}{\Psi^{0}}\left(\partial_{\alpha} T_{\mu \nu}^{0} \partial_{\alpha}+\partial_{\alpha} T_{\alpha \nu}^{0} \partial_{\mu}+\partial_{\nu} T_{\alpha \mu}^{0} \partial_{\alpha}\right) \frac{1}{\Psi^{0}} \\
& +V_{, \mu \nu}^{\mathrm{KS}}+\frac{1}{4} \frac{1}{\Psi^{0}} \partial_{\nu} \partial_{\alpha} n \partial_{\alpha} \partial_{\mu} \frac{1}{\Psi^{0}}
\end{aligned}
$$

Using the derivative operator identity

$$
\frac{1}{\Psi^{0}} \partial_{\alpha}-\partial_{\alpha} \frac{1}{\Psi^{0}}=\frac{\eta_{\alpha}}{\Psi^{0}}
$$

and defining $t_{\mu \nu}=T_{\mu \nu}^{0} / n^{0}$, allows us to convert (48) into the following succinct and symmetric reformulation

$$
\begin{aligned}
\hat{R}_{\mu \nu}^{(\boldsymbol{\xi})}= & V_{, \mu \nu}^{\mathrm{KS}}+\frac{1}{4}\left(\partial_{\nu}+\eta_{\nu}\right)\left(\nabla^{2}-S\right)\left(\partial_{\mu}-\eta_{\mu}\right) \\
& -\left(\partial_{\alpha}+\eta_{\alpha}\right) t_{\mu \nu}\left(\partial_{\alpha}-\eta_{\alpha}\right) \\
& -\left(\partial_{\alpha}+\eta_{\alpha}\right) t_{\alpha \nu}\left(\partial_{\mu}-\eta_{\mu}\right) \\
& -\left(\partial_{\nu}+\eta_{\nu}\right) t_{\alpha \mu}\left(\partial_{\alpha}-\eta_{\alpha}\right)
\end{aligned}
$$

which we can use in (44). Here

$$
\begin{aligned}
S & =\nabla^{2}-(\nabla+\boldsymbol{\eta}) \cdot(\nabla-\boldsymbol{\eta}) \\
& =\left[\partial_{\alpha} \eta_{\alpha}\right]+\eta_{\alpha} \eta_{\alpha}=\left(\left[\frac{1}{2} \nabla^{2} n^{0}\right] / n^{0}-\eta_{\alpha} \eta_{\alpha}\right) .
\end{aligned}
$$

All functions appearing in these expressions depend on groundstate orbital wavefunctions and their derivatives only. Expanding in terms of occupied orbitals they are

$$
\begin{aligned}
t_{\mu \nu} & =\frac{\Re \frac{1}{2} \sum_{i}\left(\left[\partial_{\mu} \psi_{i}^{*}\right]\left[\partial_{\nu} \psi_{i}\right]-\psi_{i}^{*}\left[\partial_{\mu} \partial_{\nu} \psi_{i}\right]\right)}{\sum_{i} \psi_{i}^{*} \psi_{i}}, \\
\eta_{\alpha} & =\frac{\Re \sum_{i} \psi_{i}^{*}\left[\partial_{\alpha} \psi_{i}\right]}{\sum_{i} \psi_{i}^{*} \psi_{i}}, \\
S & =\frac{\Re \sum_{i}\left(\left[\partial_{\alpha} \psi_{i}^{*}\right]\left[\partial_{\alpha} \psi_{i}\right]+\psi_{i}^{*}\left[\nabla^{2} \psi_{i}\right]\right)}{\sum_{i} \psi_{i}^{*} \psi_{i}}-\eta_{\alpha} \eta_{\alpha} .
\end{aligned}
$$

Here the force balance equation $V_{, \mu}^{\mathrm{KS}}=-\left(\partial_{\alpha}+2 \eta_{\alpha}\right) t_{\alpha \mu}$ replaces the usual $n^{0} V_{, \mu}^{\mathrm{KS}}=-\partial_{\alpha} T_{\alpha \mu}^{0}$. In a one-electron system with occupied orbital $\psi$ and energy $\epsilon_{0}$ these reduce to

$$
\begin{aligned}
\eta_{\alpha} & =\frac{\psi, \alpha}{\psi}, \\
S & =2\left(V^{\mathrm{KS}}-\epsilon_{0}\right) .
\end{aligned} \quad t_{\mu \nu}=\frac{1}{2}\left(\eta_{\mu} \eta_{\nu}-\frac{\psi_{, \mu \nu}}{\psi}\right),
$$

It is worth noting that, for molecular systems with Coulomb-like nuclear potentials the outermost tail is dominated by one-electron-like behaviour and:

1. The denominators of $t_{\mu \nu}, \eta_{\alpha}$ and $S$ are densities and thus everywhere positive,

2. $t_{\mu \nu}(\boldsymbol{r}) \underset{r \rightarrow \infty}{\sim} 0$,

3. $|\boldsymbol{\eta}(\boldsymbol{r})| \underset{r \rightarrow \infty}{\sim}\left|\sqrt{-2 \epsilon_{H}}\right|$ where $\epsilon_{H}$ is the KS eigenvalue of the highest occupied orbital,

4. $S(\boldsymbol{r}) \underset{r \rightarrow \infty}{\sim} 2\left(V^{\mathrm{KS}}(\boldsymbol{r})-\epsilon_{H}\right)$.

where $\boldsymbol{r}$ is the displacement from the center of the highest occupied orbital and we ignore leading terms that decay exponentially. In large molecules these expressions may also hold true closer to nucleii $A$ with $\epsilon_{H}$ replaced by the local $\epsilon_{H}^{A}$.

To reiterate, the 'orthonormal' reformulation of the QCM equations is introduced to better deal with finite systems such as atoms and molecules using common methods such as expansion on GTOs and STOs. Use of the quasi-derivatives $\partial_{\alpha} \pm \eta_{\alpha}$ makes evaluation of the normalised operator (50) fairly straightforward. Practical outputs, such as correlation energies and responses, can be obtained via the set of QCM eigenmodes $\boldsymbol{\xi}_{N}$ which asymptotically decay in finite systems. These eigen-modes are solutions of the eigen-equations $\Omega_{N}^{2} \boldsymbol{\xi}_{N}=\hat{\mathrm{R}}^{(\boldsymbol{\xi})} \boldsymbol{\xi}_{N}$ [ie. (44)] using the operator defined in (50) and are normalised via $\int \mathrm{d} \boldsymbol{r} \boldsymbol{\xi}_{N}^{*} \cdot \boldsymbol{\xi}_{N}=1$.

We will now proceed in Section $\mathrm{V}$ to investigate how this reformulation applies to one-electron systems, and through this provide proof that the QCM is exact in such systems. Using the results of Section $\mathrm{V}$ we then motivate an approximation to the QCM in Section VI, that remains exact in one-electron systems. 


\section{ONE-ELECTRON SYSTEMS}

Following equations (41) and (45-46) of Ref. 4, it is possible to show that the non-linearised QCM formalism is equivalent to Madelung hydrodynamics ${ }^{1}$ in a Lagrangian frame for one electron systems. If all external fields involve gradients of scalar potentials only, this is directly equivalent ${ }^{27}$ to finding solutions of the oneelectron, time-dependent Schrödinger equation (SE). We note that both one-electron systems, and two-electron systems with equal densities of spin up and down are covered.

Here we show this equivalence directly from the SE in the linear response limit required by the density response $\hat{\chi}_{0}$. This direct proof provides motivation for the approximation to the QCM given in the following Section VI, by ensuring it is exact in a one-electron system.

We proceed with the proof as follows: i) we derive the relationship between the perturbed one-electron wavefunction and its "orthonormal displacement vector" $\boldsymbol{\xi}^{1 e^{-}}$ to show that the latter is entirely determined by the former (and vice versa up to a trivial phase via the Runge-Gross theorem ${ }^{9}$ ); ii) we show that finding a freestanding solution of the linear-perturbed SE for a oneelectron system is equivalent to solving an equation of form $\omega^{2} \boldsymbol{\xi}^{1 e^{-}}=\hat{\mathrm{R}}^{1 e^{-}} \boldsymbol{\xi}^{1 e^{-}}$; and iii) we show that $\hat{\mathrm{R}}^{1 e^{-}}=\hat{\mathrm{R}}$ as defined in (17).

In any one-electron system we can set $V=V^{\mathrm{KS}}-\epsilon_{0}$ (noting that $V^{\mathrm{KS}}=V^{\mathrm{Ext}}$ ) to eliminate the energy of the single occupied orbital. Thus the groundstate Hamiltonian takes the form

$$
\left(-\frac{1}{2} \nabla^{2}+V\right) \psi \equiv \hat{h} \psi=0
$$

where $\psi$ is the only occupied electron wavefunction which we make real. If we apply a small, time-dependent external potential $V^{1}(t)$ then we can find a new solution $\psi^{\prime}(t)$ through

$$
\left[\hat{h}+V^{1}(t)\right] \psi^{\prime}(t)=-i \partial_{t} \psi^{\prime}(t) .
$$

which will be perturbed only slightly from the groundstate solution. We can write the perturbed wavefunction via a change to its magnitude and a rotation of its phase such that

$$
\psi^{\prime}(t)=\left[\psi+\psi^{1}(t)\right] e^{i \phi^{1}(t)}
$$

or, truncating to first order, $\psi^{\prime}(t) \approx \psi+\psi^{1}(t)+i \psi \phi^{1}(t)$. Thus it is sufficient to calculate $\phi^{1}$ and $\psi^{1}$ to fully determine the perturbed solution.

Inserting (58) into (57) and matching real and imaginary components (to linear order) gives

$$
\begin{aligned}
\psi \partial_{t} \phi^{1}(t) & =\psi V^{1}(t)+\hat{h} \psi^{1}(t), \\
\partial_{t} \psi^{1}(t) & =(\nabla \psi) \cdot\left[\nabla \phi^{1}(t)\right]+\frac{1}{2} \psi \nabla^{2} \phi^{1}(t) .
\end{aligned}
$$

where the second expression relates $\partial_{t} \psi^{1}$ directly to $\nabla \phi^{1}$. If we then assume a time-periodic external potential $V^{1}(t)=V^{1} e^{i \omega t}$ it follows that $\psi^{1}(t)=\psi^{1} e^{i \omega t}$ and $\phi^{1}(t)=\phi^{1} e^{i \omega t}$ and thus $\partial_{t} \equiv i \omega$. We can then use (60) to eliminate $\psi^{1}$ and derive the equations governing density perturbations in terms of $\phi^{1}$ only. Here

$$
\begin{aligned}
-\omega^{2} \phi^{1} & =\frac{1}{2} \psi^{-1} \hat{h}\left(\psi_{, \alpha}+\partial_{\alpha} \psi\right) \partial_{\alpha} \phi^{1}+i \omega V^{1}(t), \\
n^{1} & =2 \psi \psi^{1}=-\frac{\psi}{-i \omega}\left(\psi, \alpha+\partial_{\alpha} \psi\right) \partial_{\alpha} \phi^{1}
\end{aligned}
$$

where $n^{1}$ is the linear change in density and we have used the derivative relationship $\partial_{\alpha} \psi-\psi \partial_{\alpha} \equiv \psi, \alpha$. By the Runge-Gross theorem ${ }^{9} V^{\prime}=V+V^{1}(t)$ is a functional of the density $n^{\prime}=n^{0}+n^{1}(t)$ only in the linear response regime, and from (62) it is clear that $\psi$ and $\nabla \phi^{1}$ are sufficient to determine electronic properties.

The Schrödinger current density $\boldsymbol{j}$ of the perturbed, one-electron system is calculated through

$$
j^{1 e^{-}}=\frac{1}{2 i}\left[\psi^{\prime} \nabla \psi^{\prime *}-\psi^{\prime *} \nabla \psi^{\prime}\right] \approx-\psi^{2} \nabla \phi^{1}
$$

where we use the first-order perturbation expression $\psi^{\prime} \approx$ $\psi+\psi^{1}+i \psi \phi^{1}$ to derive the second identity. In general ${ }^{24}$ the displacement $\boldsymbol{u}$ is related to the current via (3) and thus $i \omega \boldsymbol{u}^{1 e^{-}}=-\nabla \phi^{1}$ for the one-electron system.

It then follows trivially that the normalised displacement $\boldsymbol{\xi}^{1 e^{-}}=\sqrt{n^{0}} \boldsymbol{u}^{1 e^{-}} \equiv \psi \boldsymbol{u}^{1 e^{-}}$is related to $\phi^{1}$ via

$$
\boldsymbol{\xi}^{1 e^{-}} \equiv \frac{\psi}{-i \omega} \nabla \phi^{1}
$$

and, from the Runge-Gross theorem, ${ }^{9}$ that $\phi^{1}$ can be obtained from $\boldsymbol{\xi}^{1 e^{-}}$. Using (62) the density perturbation takes the expected form (43) $n^{1}=-\nabla \cdot \psi \boldsymbol{\xi}^{1 e^{-}}$. This completes the first stage of the proof.

By taking the gradient of (61) and using $\nabla \phi^{1}=$ $(-i \omega / \psi) \boldsymbol{\xi}^{1 e^{-}}$[from $\left.(64)\right]$ we can explicitly solve the freestanding $\left(V^{1}=0\right)$ equation for $\boldsymbol{\xi}^{1 e^{-}}$via

$$
\begin{aligned}
-\omega^{2} \frac{\xi_{\mu}^{1 e^{-}}}{\psi} & =\frac{1}{2} \partial_{\mu} \psi^{-1} \hat{h}\left(\psi_{, \nu}+\partial_{\nu} \psi\right) \frac{\xi_{\nu}^{1 e^{-}}}{\psi} \\
\omega^{2} \xi_{\mu}^{1 e^{-}} & =\frac{1}{4}\left(\partial_{\mu}-\eta_{\mu}\right)\left(\nabla^{2}-S\right)\left(\partial_{\nu}+\eta_{\nu}\right) \xi_{\nu}^{1 e^{-}}
\end{aligned}
$$

where we used the one-electron specific relationships (55) $\eta_{\alpha}=\psi, \alpha / \psi$ and $2 \hat{h}=S-\nabla^{2}$ for the second expression. Thus we can define a linear operator

$$
\hat{R}_{\mu \nu}^{1 e^{-}}=\frac{1}{4}\left(\partial_{\mu}-\eta_{\mu}\right)\left(\nabla^{2}-S\right)\left(\partial_{\nu}+\eta_{\nu}\right)
$$

such that $\omega^{2} \xi_{\mu}^{1 e^{-}}=\hat{R}_{\mu \nu}^{1 e^{-}} \xi_{\nu}^{1 e^{-}}$which is of the same form as (5), as desired for the second stage of the proof. 
Finally it remains to be shown that $\hat{R}=\hat{R}^{1 e^{-}}$. Following the working in Appendix D we show that

$$
\begin{aligned}
\hat{R}_{\mu \nu}^{1 e^{-}}= & V_{, \mu \nu}+\frac{1}{4}\left(\partial_{\nu}+\eta_{\nu}\right)\left(\nabla^{2}-S\right)\left(\partial_{\mu}-\eta_{\mu}\right) \\
& -\left(\partial_{\alpha}+\eta_{\alpha}\right) t_{\mu \nu}\left(\partial_{\alpha}-\eta_{\alpha}\right) \\
& -\left(\partial_{\alpha}+\eta_{\alpha}\right) t_{\alpha \nu}\left(\partial_{\mu}-\eta_{\mu}\right) \\
& -\left(\partial_{\nu}+\eta_{\nu}\right) t_{\alpha \mu}\left(\partial_{\alpha}-\eta_{\alpha}\right) \\
\equiv & \hat{R}_{\mu \nu}^{(\xi)}
\end{aligned}
$$

and the proof is complete.

Thus we have shown that i) $\boldsymbol{\xi}^{1 e^{-}}$is bijectively (up to a phase) related to the first-order solution of the perturbed $\mathrm{SE}$; ii) It obeys $\omega^{2} \boldsymbol{\xi}^{1 e^{-}}=\hat{\mathrm{R}}^{1 e^{-}} \boldsymbol{\xi}^{1 e^{-}}$for free-standing modes and iii) $\hat{\mathrm{R}}^{1 e^{-}}=\hat{\mathrm{R}}$. Thus the governing equation is identical in both cases and it follows that $\xi^{1 e^{-}} \equiv \boldsymbol{\xi}$. This confirms that the solutions of the QCM equations are directly equivalent to the solutions of the perturbed Schrödinger equation in one-electron systems, and that the QCM is thus exact.

\section{A. Transition modes of one-electron systems}

In a one-electron system, the density response $\hat{\chi}_{0}$ calculated via orbital transitions (29) must be the same as that caclulated via the QCM transitions(27). Thus

$$
\chi_{0}=\sum_{a} \frac{d_{a 0}(\boldsymbol{r}) d_{a 0}\left(\boldsymbol{r}^{\prime}\right)}{\Omega_{a 0}^{2}+\sigma^{2}}=\sum_{N} \frac{d_{N}(\boldsymbol{r}) d_{N}\left(\boldsymbol{r}^{\prime}\right)}{\Omega_{N}^{2}+\sigma^{2}} .
$$

where $a$ is summed over the unoccopied orbitals, while $\Omega_{a 0}=\epsilon_{a}-\epsilon_{0}$ and $d_{a 0}= \pm \sqrt{2 \Omega_{a 0}} \psi \psi_{a}$. Since the equality must be true for all $\sigma$ it follows that the individual numerators and denominators of the sums must be paired and we can choose an $N_{a}$ such that $\Omega_{N_{a}}=\Omega_{a 0}$ and $d_{N_{a}}(\boldsymbol{r})=d_{a 0}(\boldsymbol{r})$.

Equating (46) and (30) gives

$$
d_{N_{a}}=-\psi(\nabla+\boldsymbol{\eta}) \cdot \boldsymbol{\xi}_{N_{a}}=\sqrt{2 \Omega_{a 0}} \psi \psi_{a} .
$$

This has a (non-unique) solution

$$
\boldsymbol{\xi}_{N_{a}}=\frac{1}{\sqrt{2 \Omega_{a 0}}}(\nabla-\boldsymbol{\eta}) \psi_{a}
$$

which must therefore be a valid solution of the QCM equations. The constant pre-factor comes from $-(\nabla+$ $\boldsymbol{\eta}) \cdot(\nabla-\boldsymbol{\eta}) \equiv 2\left(\hat{h}-\epsilon_{0}\right)$ in a one-electron system.

Tao et $a l^{5}$ tested the one-electron exactness on general $s$-transitions in the Hydrogen atom. We illustrate (70) and $(71)$ on the $1 s$ to $2 p$ transition. Here the $1 s$ orbital is occupied with orbital $\psi_{1 s}=e^{-r} / \sqrt{\pi}$ and energy $\epsilon_{0}=$ $\epsilon_{1 s}=-\frac{1}{2}$ while the $2 p_{z}$ orbital has $\psi_{2 p_{z}}=z e^{-r / 2} / \sqrt{32 \pi}$ and $\epsilon_{2 p_{z}}=-\frac{1}{8}$. The density is thus $n^{0}=e^{-2 r} / \pi$ and $\boldsymbol{\eta}=-\hat{\boldsymbol{r}}$.
Using $\nabla z e^{-r / 2}=\left(\hat{\boldsymbol{z}}-\frac{1}{2} z \hat{\boldsymbol{r}}\right) e^{-r / 2}$ we can test $(70)$ and (71). With $\Omega_{2 p_{z}-1 s}=\frac{3}{8}$ we find

$$
\begin{aligned}
\boldsymbol{\xi}_{2 p_{z}-1 s} & =\frac{1}{\sqrt{96 \pi}} e^{-r / 2}(z \hat{\boldsymbol{r}}+2 \hat{\boldsymbol{z}}) \\
d_{2 p_{z}-1 s} & =-\frac{\psi_{1 s}}{\sqrt{96 \pi}}(\nabla-\hat{\boldsymbol{r}}) \cdot(z \hat{\boldsymbol{r}}+2 \hat{\boldsymbol{z}}) e^{-r / 2} \\
& =\sqrt{\frac{3}{4}} \psi_{1 s} \psi_{2 p_{z}} .
\end{aligned}
$$

in agreement with (70) and where $3 / 4=2 \Omega_{1 s-2 p_{z}}$ as expected. Note that the usual displacement vector $\boldsymbol{u}_{2 p_{z}-1 s}=\frac{e^{r / 2}}{\sqrt{96 \pi}}(z \hat{\boldsymbol{r}}+2 \hat{\boldsymbol{z}})$ grows exponentially with $r$.

We have thus shown that, in one-electron systems, the QCM is exact. A one-to-one relationship between the QCM density modes and KS transition densities [Equation 71] is also established which we will use as motivation for an approximation.

\section{SCALAR APPROXIMATION TO QCM}

While the exact properties of one-electron systems will not hold true in general, they do suggest a way to approximate the QCM in such a way that it remains exact for one-electron systems. This is done by using the form of the one-electron QCM density modes defined in (71), to approximately expand the density modes in general, many-electron systems. Such an approach would not be expected to provide accurate vector/tensor properties (such as the tensor response) but might prove acceptable for scalar properties (such as the scalar density response).

To begin with we note that any well-behaved vector function can be written as a gradient plus a curl. We may thus set

$$
\boldsymbol{\xi}_{N}(\boldsymbol{r})=\Psi^{0}\left\{\nabla s_{N}(\boldsymbol{r})-\nabla \times \boldsymbol{v}_{N}(\boldsymbol{r})\right\}
$$

where $s_{N}(\boldsymbol{r})$ and $\boldsymbol{v}_{N}(\boldsymbol{r})$ are arbitrary scalar/vector functions with appropriate asymptotes (we choose our gauge to ensure $\nabla \cdot \boldsymbol{v}_{N}=0$ ). The form of (71) suggests that we might approximate our eigen-solutions $\boldsymbol{\xi}_{N}$ by setting

$$
\tilde{\boldsymbol{\xi}}_{N}(\boldsymbol{r}) \approx\{\nabla-\boldsymbol{\eta}(\boldsymbol{r})\} \phi_{N}(\boldsymbol{r})=\Psi^{0}(\boldsymbol{r}) \nabla \frac{\phi_{N}(\boldsymbol{r})}{\Psi^{0}(\boldsymbol{r})}
$$

which is equivalent to making the approximation $s_{N} \approx$ $\phi_{N} / \Psi^{0}$ and $\boldsymbol{v}_{N} \approx \mathbf{0}$. This approximation will be exact for one-electron systems and one-dimensional (1D) systems $^{28}$ but is not true in general.

The regular QCM eigen-equation (44) is equivalent to finding stationary solutions $\delta \mathcal{E} / \delta \boldsymbol{\xi}=\mathbf{0}$ of $\mathcal{E}[\boldsymbol{\xi}]=$ $\frac{1}{2} \int \mathrm{d} \boldsymbol{r} \boldsymbol{\xi}^{*} \cdot \hat{\mathrm{R}} \cdot \boldsymbol{\xi}$ for vectors $\boldsymbol{\xi}$ satisfying $\int \mathrm{d} \boldsymbol{r} \boldsymbol{\xi}^{*} \cdot \boldsymbol{\xi}=1$. We called these solutions $\boldsymbol{\xi}_{N}$ and they can be found through $\Omega_{N}^{2} \boldsymbol{\xi}_{N}=\hat{\mathrm{R}} \boldsymbol{\xi}_{N}$ where $\hat{\mathrm{R}}$ is defined in (17). Under the scalar approximation we restrict our solutions to vectors $\tilde{\boldsymbol{\xi}}$ expressible as $(\nabla-\boldsymbol{\eta}) \phi$ which form a connected 
subspace of all possible $\boldsymbol{\xi}$. We thus look for solutions $\tilde{\boldsymbol{\xi}}_{N}(\phi)$ of $\mathcal{E}[\boldsymbol{\xi}]$ which are stationary under variation of $\phi$ ie. $\delta \mathcal{E}[\boldsymbol{\xi}(\phi)] / \delta \phi=0$ subject to $\int \mathrm{d} \boldsymbol{r} \tilde{\boldsymbol{\xi}}(\phi) \cdot \tilde{\boldsymbol{\xi}}(\phi)=1$.

The restricted solutions can be found directly by setting $\tilde{\mathcal{E}}[\phi]=-\frac{1}{2} \int \mathrm{d} \boldsymbol{r} \phi^{*}(\nabla+\boldsymbol{\eta}) \cdot \hat{\mathrm{R}} \cdot(\nabla-\boldsymbol{\eta}) \phi$ and setting the constraint to $-\int \mathrm{d} \boldsymbol{r} \phi^{*}(\nabla+\boldsymbol{\eta}) \cdot(\nabla-\boldsymbol{\eta}) \phi=1$. The general Hermitian eigen-equation for $\phi_{N}$ thus becomes

$$
\tilde{\Omega}_{N}^{2} \hat{N}^{\phi} \phi_{N}=\hat{R}^{\phi} \phi_{N}
$$

with orthogonal solutions normalised under $\int \mathrm{d} \boldsymbol{r} \phi_{N}^{*} \hat{N}^{\phi} \phi_{M}=\delta_{N M}$. Here

$$
\begin{aligned}
\hat{N}^{\phi} & =-(\nabla+\boldsymbol{\eta}) \cdot(\nabla-\boldsymbol{\eta})=S-\nabla^{2} \\
\hat{R}^{\phi} & =-\left(\partial_{\mu}+\eta_{\mu}\right) \hat{R}_{\mu \nu}\left(\partial_{\nu}-\eta_{\nu}\right) .
\end{aligned}
$$

It is obvious that $\tilde{\Omega}_{1}^{2} \geq \Omega_{1}^{2}$ since the subspace minimum of $\mathcal{E}$ must be equal to or higher than its true minimum.

Such an approximation loses some accuracy and some nice properties of the true QCM but reduces the problem from a tensor to a scalar. Its exactness in a variety of systems including the one-electron case in any number of dimensions suggests that it might be appropriate for $\mathrm{vdW}$ calculations in molecular systems. Here the vdW physics are often dominated by the asymptotic regions which show one-electron-like behaviour (see Section VIC for further details).

In the remaining subsections we will investigate some of the practical results of this approximation. These are not intended to be a thorough investigation of the method, but to provide some guidance to the numerical and theoretical analysis thereof.

\section{A. Scalar approximation in KS orbitals}

We can expand $\phi_{N}=\sum_{j} p_{N j} \psi_{j}$ in the KS orbitals (or any other complete and orthonormal basis set) so that, from (75),

$$
\tilde{\boldsymbol{\xi}}_{N}(\boldsymbol{r})=\sum_{j} p_{N j}\{\nabla-\boldsymbol{\eta}(\boldsymbol{r})\} \psi_{j}(\boldsymbol{r})
$$

where $\psi_{j}$ are KS orbitals (occupied or otherwise). In reality we must truncate to the lowest $N_{\text {Bas }}$ orbitals. The transition density modes are thus (remembering that $\left.\sqrt{n^{0}}=\Psi^{0}\right)$

$$
\begin{aligned}
\tilde{d}_{N}(\boldsymbol{r}) & =-\Psi^{0} \sum_{j} p_{N j}(\nabla+\boldsymbol{\eta}) \cdot(\nabla-\boldsymbol{\eta}) \psi_{j} \\
& =\Psi^{0} \sum_{j} p_{N j}\left(S-\nabla^{2}\right) \psi_{j} \\
& =\Psi^{0} \sum_{j} p_{N j}\left[2\left(\epsilon_{j}-V^{\mathrm{KS}}\right)+S\right] \psi_{j} .
\end{aligned}
$$

where the first two properties are true for any basis set but the third property is only true for the KS orbitals.
Projection into the KS orbitals allows some insight into the physical meaning of this approximation to be obtained by considering the quasi-orbital $\Psi^{0}$. Here we can set $\tilde{\boldsymbol{\xi}}_{N}=\left(2 i / \Psi^{0}\right) \sum_{j} p_{N j} \boldsymbol{j}_{j}$ where the quasi-transition current

$$
\boldsymbol{j}_{j}=\frac{1}{2 i}\left[\Psi^{0}(\nabla-\boldsymbol{\eta}) \psi_{j}\right]=\frac{1}{2 i}\left[\Psi^{0} \nabla \psi_{j}-\psi_{j} \nabla \Psi^{0}\right]
$$

has a similar form to a transition current $\boldsymbol{j}_{a i}=$ $\frac{1}{2 i}\left(\psi_{i} \nabla \psi_{a}-\psi_{a} \nabla \psi_{i}\right)$ with the occupied orbital replaced by the quasi-orbital of the total density.

If we pre-multiply (44) by $\tilde{\boldsymbol{\xi}}_{M}$ and integrate we find $\tilde{\Omega}_{N}^{2} \int \mathrm{d} \boldsymbol{r} \tilde{\boldsymbol{\xi}}_{M} \cdot \tilde{\boldsymbol{\xi}}_{N}=\int \mathrm{d} \boldsymbol{r} \tilde{\boldsymbol{\xi}}_{M} \hat{\mathrm{R}} \tilde{\boldsymbol{\xi}}_{N}$ subject to $\int \mathrm{d} \boldsymbol{r} \tilde{\boldsymbol{\xi}}_{M} \cdot \tilde{\boldsymbol{\xi}}_{N}=$ $\delta_{M N}$. Using (79) the two integrals become

$$
\begin{aligned}
\int \mathrm{d} \boldsymbol{r} \tilde{\boldsymbol{\xi}}_{M}^{*} \cdot \tilde{\boldsymbol{\xi}}_{N} & =\sum_{j k} p_{M j}^{*} p_{N k} N_{j k}, \\
\int \mathrm{d} \boldsymbol{r} \tilde{\boldsymbol{\xi}}_{M}^{*} \hat{\mathrm{R}} \tilde{\boldsymbol{\xi}}_{N} & =\sum_{j k} p_{M j}^{*} p_{N k} R_{j k} .
\end{aligned}
$$

where

$$
\begin{aligned}
& N_{j k}=\int \mathrm{d} \boldsymbol{r}\left[\left(\partial_{\alpha}-\eta_{\alpha}\right) \psi_{j}^{*}\right]\left[\left(\partial_{\alpha}-\eta_{\alpha}\right) \psi_{k}\right], \\
& R_{j k}=\int \mathrm{d} \boldsymbol{r}\left[\left(\partial_{\mu}-\eta_{\mu}\right) \psi_{j}^{*}\right] \hat{R}_{\mu \nu}\left[\left(\partial_{\nu}-\eta_{\nu}\right) \psi_{k}\right] .
\end{aligned}
$$

Minimising with respect to $p_{M j}$, the eigen-equation (44) thus becomes

$$
\tilde{\Omega}_{N}^{2} N_{j k} p_{N k}=R_{j k} p_{N k}
$$

subject to the orthogonality condition $\sum_{j k} N_{j k} p_{M j}^{*} p_{N k}=\delta_{N M}$. Following the details of Appendix E we find

$$
\begin{aligned}
R_{j k}= & \int \mathrm{d} \boldsymbol{r}\left[\hat{D}_{\mu} \psi_{j}^{*}\right] V_{, \mu \nu}\left[\hat{D}_{\nu} \psi_{k}\right] \\
& +\frac{1}{4} \int \mathrm{d} \boldsymbol{r}\left[\hat{D}_{\alpha} \hat{D}_{\mu} \hat{D}_{\nu} \psi_{j}^{*}\right]\left[\hat{D}_{\alpha} \hat{D}_{\mu} \hat{D}_{\nu} \psi_{k}\right] \\
& +3 \int \mathrm{d} \boldsymbol{r}\left[\hat{D}_{\alpha} \hat{D}_{\mu} \psi_{j}^{*}\right] t_{\mu \nu}\left[\hat{D}_{\alpha} \hat{D}_{\nu} \psi_{k}\right]
\end{aligned}
$$

where $\hat{D}_{\alpha} \equiv \partial_{\alpha}-\eta_{\alpha}$.

\section{B. Matrix form of the scalar approximation}

Since all terms involve repeated use of the operator $\left(\partial_{\alpha}-\eta_{\alpha}\right)$ we may simplify things somewhat by adopting a matrix notation to represent this operator. For a finite basis set of size $N_{\text {Bas }}$ we define the following $N_{\text {Bas }} \times N_{\text {Bas }}$ matrices: $\mathbb{D}_{\alpha}, \mathbb{T}_{\mu \nu}$ and $\mathbb{V}_{\mu \nu}$ with elements

$$
\begin{aligned}
D_{\alpha j k} & =\int \mathrm{d} \boldsymbol{r} \psi_{j}^{*}\left(\partial_{\alpha}-\eta_{\alpha}\right) \psi_{k}, \\
T_{\mu \nu j k} & =\int \mathrm{d} \boldsymbol{r} \psi_{j}^{*} t_{\mu \nu} \psi_{k}, \\
V_{\mu \nu j k} & =\int \mathrm{d} \boldsymbol{r} \psi_{j}^{*} V_{, \mu \nu}^{\mathrm{KS}} \psi_{k} .
\end{aligned}
$$


We can also integrate by parts to obtain $V_{\mu \nu j k}=$ $\int \mathrm{d} \boldsymbol{r} V^{\mathrm{KS}}\left[\partial_{\mu} \partial_{\nu} \psi_{j}^{*} \psi_{k}\right]$. Noting that the orbitals $\psi_{j}$ form an orthonormal set we see that $\left(\partial_{\alpha}-\eta_{\alpha}\right) \psi_{k}=\sum D_{\alpha j k} \psi_{j}$ with similar relationships for the others.

This allows us to write the matrix eigen-equation

$$
\tilde{\Omega}_{N}^{2} \mathbb{N P}_{N}=\mathbb{R} \mathbb{P}_{N}
$$

where $\mathbb{P}_{N}$ is an $N_{\text {Bas }} \times 1$ matrix with elements $p_{N j}$. Here

$$
\begin{aligned}
& \mathbb{N}=\mathbb{D}_{\alpha}^{\dagger} \mathbb{D}_{\alpha} \\
& \mathbb{R}=\mathbb{D}_{\mu}^{\dagger} \mathbb{V}_{\mu \nu} \mathbb{D}_{\nu}+\mathbb{D}_{\mu}^{\dagger} \mathbb{D}_{\alpha}^{\dagger}\left(3 \mathbb{T}_{\mu \nu}+\frac{1}{4} \mathbb{D}_{\nu}^{\dagger} \mathbb{D}_{\mu}\right) \mathbb{D}_{\alpha} \mathbb{D}_{\nu}
\end{aligned}
$$

Orthogonality is given by $\mathbb{P}_{N}^{\dagger} \mathbb{N P}_{M}=\delta_{N M}$. We note that all tensor components are bundled into $\mathbb{N}$ and $\mathbb{R}$ via summation over $\alpha, \mu$ and $\nu$. These equations are only true in the strict limit of an infinite number of orbitals but will converge to the true approximate solution in typical systems.

\section{One-electron like asymptotic behaviour}

In the asymptotic $|\boldsymbol{r}| \rightarrow \infty$ region of a localised system, the highest occupied molecular orbital (HOMO) $\psi_{H}$ with occupation number $f_{H}$ dominates [at least in the non-degenerate case or for degenerate system with spherical symmetry where we set $f_{H}=\left(2 l_{H}+1\right)$ and $\psi_{H}(\boldsymbol{r})=R_{n_{H}}(r)$, the radial component of $\psi_{n_{H} l_{H} m}(\boldsymbol{r})=$ $\left.R_{n_{H}}(r) Y_{l_{H} m}\left(\Omega_{r}\right)\right]$. Thus, in the limit $\boldsymbol{r} \rightarrow \infty$ we can set:

$$
\begin{aligned}
n^{0} \approx f_{H}\left|\psi_{H}\right|^{2}, & \boldsymbol{\eta} \approx \frac{\left[\nabla \psi_{H}\right]}{\psi_{H}}, \\
t_{\mu \nu} \approx-\frac{1}{2} \partial_{\mu} \eta_{\nu}, & S \approx 2\left(V^{\mathrm{KS}}-\epsilon_{H}\right) .
\end{aligned}
$$

All equations reduce to the one-electron form in these limits, with the single occupied orbital given by the HOMO.

We can check how close a region of a many-electron system is to a one-electron-like system by considering the difference of $S$ from its asymptotic form. Here we set

$$
\begin{aligned}
\tilde{S}(\boldsymbol{r}) & =S(\boldsymbol{r})-2\left(V^{\mathrm{KS}}(\boldsymbol{r})-\epsilon_{H}\right) \\
& =C^{0}(\boldsymbol{r})+2\left(\epsilon_{H}-\tilde{\epsilon}(\boldsymbol{r})\right)
\end{aligned}
$$

where

$$
\begin{aligned}
C^{0}(\boldsymbol{r}) & =\sum_{i} \frac{f_{i}\left|\nabla \psi_{i}(\boldsymbol{r})\right|^{2}}{n^{0}(\boldsymbol{r})}-|\boldsymbol{\eta}(\boldsymbol{r})|^{2} \\
\tilde{\epsilon}(\boldsymbol{r}) & =\sum_{i} \frac{f_{i}\left|\psi_{i}(\boldsymbol{r})^{2}\right|}{n^{0}(\boldsymbol{r})} \epsilon_{i}
\end{aligned}
$$

and where $C^{0}$ is related to the Fermi-hole curvature ${ }^{29}$ of the KS system. In the limit $|\boldsymbol{r}| \rightarrow \infty$ it is clear that $C^{0}(\boldsymbol{r})=0$ and $\tilde{\epsilon}(\boldsymbol{r})=\epsilon_{H}$ (true everywhere for oneelectron systems).
This outer region is crucial for van der Waals physics in many systems. Typically only transitions within a small frequency range, especially that to the lowest unoccupied molecular orbital (LUMO), will dominate in this region. In such transitions $\int \mathrm{d} \boldsymbol{r} \psi_{k}^{*} \tilde{S} \psi_{k}$ will be small. The asymptotic 'exactness' in this limit suggest strongly that the scalar approximation to $\mathrm{QCM}$ is a appropriate for calculating vdW forces.

Even a well chosen but simple approximation, using a limited number of unoccupied orbitals and quasiorbitals, may provide quite accurate estimations of the vdW physics. The ability of the QCM (and the scalar approcimation) to make collective modes for the transition physics may aid in convergence compared to full dRPA calculations.

\section{Transitions and energy calculations in the scalar approximation}

Once we have obtained solutions of (91) we can use $p_{N j}$ to evaluate $d_{N}$ through (80). Using $\tilde{\Omega}_{N}$ also we can then evaluate $\hat{\chi}_{0}$ and properties which depend on it such as the correlation energy.

We can also use the asymptotic form of $S(\boldsymbol{r})$ to further investigate $d_{N}$ and through it correlations. Combining (80) and (96) lets us write

$$
\begin{aligned}
d_{N} & =\Psi^{0} \sum_{j} p_{N j}\left[2\left(V^{\mathrm{KS}}-\epsilon_{H}\right)-\nabla^{2}+\tilde{S}\right] \psi_{j} \\
& =\Psi^{0} \sum_{j} p_{N j}\left(2 \Omega_{j H}+\tilde{S}\right) \psi_{j}
\end{aligned}
$$

where the $\Omega_{j H}=\epsilon_{j}-\epsilon_{H}$ comes from applying the Schrödinger Hamiltonian $-\frac{1}{2} \nabla^{2}+V^{\mathrm{KS}}$ to $\psi_{j}$. Here $\tilde{S}$ covers the deviation of many-electron systems from their one-electron equivalents.

Let us expand (99) as follows:

$$
d_{N}=\Psi^{0} \sum_{j} d_{N j} \psi_{j}
$$

We define matrices $\mathbb{O}$ with $O_{j k}=\delta_{j k} \Omega_{k H}$ and $\tilde{\mathbb{S}}$ with $\tilde{S}_{j k}=\int \mathrm{d} \boldsymbol{r} \psi_{j}^{*} \tilde{S} \psi_{k}$. Thus $d_{N j}=\left[\mathbb{D}_{N}\right]_{j}$ where $\mathbb{D}_{N}=$ $(2 \mathbb{O}+\tilde{\mathbb{S}}) \mathbb{P}_{N}$ and $\mathbb{P}_{N}$ is a solution of $(91)$. We can use this expansion to calculate the Coulomb projections $W_{N M}$ defined in (37). Using (100) we find

$$
\begin{aligned}
W_{N M} & =\mathbb{D}_{M}^{\dagger} \mathbb{W}^{(\psi)} \mathbb{D}_{N} \\
& =\mathbb{P}_{M}^{\dagger}(2 \mathbb{O}+\tilde{\mathbb{S}}) \mathbb{W}^{(\psi)}(2 \mathbb{O}+\tilde{\mathbb{S}}) \mathbb{P}_{N}
\end{aligned}
$$

where

$$
\left[\mathbb{W}^{(\psi)}\right]_{j k}=\int \mathrm{d} \boldsymbol{r} \mathrm{d} \boldsymbol{r} \frac{\Psi^{0}(\boldsymbol{r}) \psi_{j}^{*}(\boldsymbol{r}) \Psi^{0}\left(\boldsymbol{r}^{\prime}\right) \psi_{k}\left(\boldsymbol{r}^{\prime}\right)}{\left|\boldsymbol{r}-\boldsymbol{r}^{\prime}\right|} .
$$

We can use $W_{N M}$ to calculate the correlation energy through equation (38). 
In molecular orbital language it is clear that the calculation of $\mathbb{W}^{(\psi)}$ is the only step involving four-center integrals required by a correlation energy calculation. However, in contrast to the four orbital (two occupied, two unoccupied) terms $(i a \mid j b)$ required by a full KS calculation it requires two uncontracted orbital indices (occupied or unuccopied) only, provided we can efficiently and accurately project $\Psi^{0}=\sqrt{n^{0}}$ onto a fitting basis set. For large molecules with many occupied orbitals this is a substantial saving.

\section{1D HARMONIC OSCILLATOR}

To illustrate the approaches discussed here, let us investigate the case of a one-dimensional (1D) manyelectron Harmonic oscillator without interactions in the groundstate. The system is thus defined as having $N_{e}$ electrons in the groundstate and $V^{\mathrm{KS}}(x)=x^{2} / 2$. Here the $\mathrm{KS}$ orbital wavefunctions and energies take the form

$$
\psi_{j}(x)=\kappa_{j} H_{j}(x) e^{-x^{2} / 2}, \quad \epsilon_{j}=\frac{2 j+1}{2}
$$

where $H_{j}(x)$ are Hermite polynomials with $j \geq 0$ and $\kappa_{j}=\left[\sqrt{\pi} 2^{j} j !\right]^{-1 / 2}$. Since the orbitals are filled up to $j \leq N_{e}-1$ the density is

$$
n^{0}(x)=\sum_{j=0}^{N_{e}-1} \frac{H_{j}(x)^{2} e^{-x^{2}}}{\sqrt{\pi} 2^{j} j !}
$$

and it is clear from the expansion of $H_{N_{e}-1}$ that $\lim _{x \rightarrow \infty} n^{0}(x) \sim x^{2\left(N_{e}-1\right)} e^{-x^{2}}$ and $\lim _{x \rightarrow \infty} \eta(x) \sim-x$. Thus the system is well-bounded and the KS-orbital approach should work well.

In the one-electron case $\eta=-x, t=\frac{1}{2}$ and $V_{, x x}=1$. and we can solve everything analytically. The matrices required to form $\mathbb{N}$ and $\mathbb{R}$ in equations (92) and (93) have elements $T_{j k}=\frac{1}{2} \delta_{j k}, V_{j k}=\delta_{j k}$ and

$$
\begin{aligned}
D_{j k} & =\kappa_{j} \kappa_{k} \int \mathrm{d} x e^{-x^{2} / 2} H_{j}(x)\left[\left(\partial_{x}+x\right) H_{k}(x) e^{-x^{2} / 2}\right] \\
& =2 k \kappa_{j} \kappa_{k} \int \mathrm{d} x e^{-x^{2}} H_{j}(x) H_{k-1}(x) \\
& =\sqrt{2(j+1)} \delta_{j(k-1)} .
\end{aligned}
$$

Here $\left[\mathbb{D}^{\dagger} \mathbb{D}\right]_{j k}=2 j \delta_{j k},\left[\mathbb{D}^{\dagger 2} \mathbb{D}^{2}\right]_{j k}=4 j(j-1) \delta_{j k}$ and $\left[\mathbb{D}^{\dagger 3} \mathbb{D}^{3}\right]_{j k}=8 j(j-1)(j-2) \delta_{j k}$.

Thus $N_{j k}=2 j \delta_{j k}$ and $R_{j k}=2 j \delta_{j k}+6 j(j-1) \delta_{j k}+$ $2 j(j-1)(j-2) \delta_{j k}=2 j^{3} \delta_{j k}$. We can ignore the $j=0$ solution as it will not contribute to $\hat{\chi}_{0}$. We thus choose solutions with $N_{j}>0$ where $p_{N_{j} j}=\delta_{N_{j} j} / \sqrt{2 j}$. Here $\Omega_{N_{j}}=\frac{2 j+1}{2}-\frac{1}{2}=j$ as expected and the transition density mode is $d_{N_{j}}(x)=-\psi_{0}(x)\left[\left(\partial_{x}^{2}+1-x^{2}\right) \psi_{j}(x)\right] / \sqrt{2 j}=$ $\sqrt{2 \Omega_{N_{j}}} \psi_{0}(x) \psi_{j}(x)$.

In the many-electron case we can solve the problem semi-analytically for much of it, requiring numerics only for terms involving $1 / n^{0}$ and subsequent diagonalisations. Errors occur due to truncation of the basis set but integrals can be obtained with near exact accuracy with Gauss-Hermite quadrature. It should be noted that the two-electron system is not predicted exactly by the QCM as there is no spin degeneracy.

The rapid convergence of the method is demonstrated in Table I where we show the convergence of the fourth transition frequency $\Omega_{4}$ for the two-electron system. With as few as 15 states the error is already under one part in ten thousand.

\begin{tabular}{|c|rrrrrr|}
\hline$N_{\text {Bas }}$ & 5 & 10 & 15 & 20 & 50 & $\infty$ \\
\hline$\Omega_{4}$ & 4.1105 & 3.8797 & 3.8805 & 3.8801 & 3.8802 & 3.8802 \\
$\log _{10}|\operatorname{Err}|$ & -1.22 & -3.95 & -4.05 & -5.06 & -7.49 & $-\infty$ \\
\hline
\end{tabular}

TABLE I. Convergence of the fourth transition frequency for the two electron system. Here Err $=\Omega_{4}^{\mathrm{N}_{\mathrm{Bas}}} / \Omega_{4}^{\infty}-1$.

The KS transition frequencies of an $N_{e}$ electron system have energies $\Omega_{a i}=\frac{2 a+1}{2}-\frac{2 i+1}{2}=J$ and each frequency $J$ has multiple contributing transitions. Here $\min \left(J, N_{e}\right)$ modes have transition frequency $J$ and the density transitions are proportional to $\psi_{i}(x) \psi_{i+J}(x)$ when $i \leq N_{e}$ and $i+J>N_{e}$.

We present the QCM transition frequencies in Table II for systems with up to 20 electrons. The QCM has singlevalued frequencies distributed approximately with the integers. Each QCM transition density is therefore composed of multiple KS-like transitions which, by the sum rules (33) must have their weights $\left|K_{a i N}\right|^{2}$ dominated by transitions with $\Omega_{a i} \approx \Omega_{N}$. In Table III we show some weights $\left|K_{a i N}\right|^{2}$ for the five electron system. It is clear that the $N$ th QCM transition puts most weight on the $\mathrm{HOMO}-\mathrm{HOMO}+N[5-(5+N)] \mathrm{KS}$ transition as one would hope.

\begin{tabular}{|rrrrrr|}
\hline \multicolumn{5}{c|}{ Transition frequencies $\Omega_{N}$} \\
$N$ & $N_{e}=1$ & $N_{e}=2$ & $N_{e}=5$ & $N_{e}=10$ & $N_{e}=20$ \\
\hline 1 & 1.0000 & 1.0000 & 1.0000 & 1.0000 & 1.0000 \\
2 & 2.0000 & 2.0000 & 2.0000 & 2.0000 & 2.0000 \\
3 & 3.0000 & 3.0000 & 3.0000 & 3.0000 & 3.0000 \\
4 & 4.0000 & 3.8802 & 3.9531 & 3.9859 & 3.9963 \\
5 & 5.0000 & 4.8680 & 4.8162 & 4.9225 & 4.9772 \\
6 & 6.0000 & 5.7877 & 5.6869 & 5.7886 & 5.9216 \\
7 & 7.0000 & 6.7689 & 6.6309 & 6.6381 & 6.8128 \\
8 & 8.0000 & 7.7154 & 7.5079 & 7.5381 & 7.6683 \\
10 & 10.0000 & 9.6579 & 9.3578 & 9.3030 & 9.4403 \\
20 & 20.0000 & 19.4882 & 18.6483 & 18.2017 & 18.1953 \\
\hline
\end{tabular}

TABLE II. First eight, 10th and 20th distinct eigenfrequencies of the KS system and QCM system with different numbers of electrons $N_{e}$. The KS transitions are all integer and degenerate with $\min \left(\Omega, N_{e}\right)$ transitions for a given integer frequency.

It is remarkable that the lowest three frequencies are exact to four decimal places for $N_{e}>1$, particularly as 
the second smallest is comprised of two transitions and the third of three in all but the two-electron case. By the sum rules (33) this means that each mode must be comprised solely of transitions with the given frequency, and as a consequence, both $\Omega_{N}$ and $d_{N}$ are predicted exactly for the HOMO-LUMO transition. That such a relationship holds for as many as 20 electrons demonstrates a strength of the physics and a resilience of the approximations in the QCM approach.

\section{CONCLUSION}

In this paper we have reformulated, simplified and investigated the QCM formalism of Refs 4-7, and provided a direct proof of its exactness in one-electron systems.

Firstly, in Section II we provide a more comprehensive derivation of, and investigation into the compact form of the QCM equations, especially with regards to the kinetic stress tensor defined by (12). The simplified QCM equation is described in (16) and surrounding work. We then discuss the QCM response function [(27) in Section III] and use it to derive a simple expression for the QCM-dRPA correlation energy in (39).

The orthonormal form described in Section IV is vital for the description and calculation of localised systems, where the standard $\boldsymbol{u}$-based formulation does not behave well. This reformulation can be written in a straightforward manner [see especially (50)] and is required for calculation of atomic, and molecular systems using standard basis set approaches involving GTO and STOs. It also provides a relatively simple way of proving (in Section $\mathrm{V}$ ), direct from the Schrödinger equation, that the QCM is exact for one-electron systems.

The scalar approximation of Section VI is then derived

\begin{tabular}{|c|c|c|c|c|c|c|c|}
\hline$N$ & $\Omega_{N}$ & $(i, a)$ & $\left|K_{a i N}^{2}\right|$ & $(i, a)$ & $\left|K_{a i N}^{2}\right|$ & $(i, a)$ & $\left|K_{a i N}^{2}\right|$ \\
\hline 1 & 1.0000 & $(5,6)$ & 100.0 & & & & \\
\hline 2 & 2.0000 & $(5,7)$ & 60.0 & $(4,6)$ & 40.0 & & \\
\hline 3 & 3.0000 & $(5,8)$ & 53.8 & $(4,7)$ & 30.8 & $(3,6)$ & 15.4 \\
\hline \multirow[t]{2}{*}{4} & 3.9531 & $(5,9)$ & 54.4 & $(4,8)$ & 27.1 & $(3,7)$ & 11.5 \\
\hline & & $(2,6)$ & 3.8 & $(4$, & 1.9 & $(5,7)$ & 1.2 \\
\hline \multirow[t]{2}{*}{5} & 4.8162 & $(5,10)$ & 52.8 & $(4,9)$ & 23.9 & $(3,8)$ & 9.1 \\
\hline & & $(3,6)$ & 6.2 & $(5,8)$ & 4.2 & $(2,7)$ & 2. \\
\hline \multirow[t]{3}{*}{7} & 6.6309 & $(5,12)$ & 49.8 & $(4,11)$ & 20.0 & $(5,10)$ & 6. \\
\hline & & $(3,10)$ & 6.7 & $(3,8)$ & 5.7 & $(2,7)$ & 5. \\
\hline & & $(1,6)$ & 1.9 & $(2,9)$ & 1.7 & & \\
\hline \multirow[t]{4}{*}{10} & 9.3578 & $(5,15)$ & 45.0 & $(4,14)$ & 16.5 & $(5,13)$ & 14.2 \\
\hline & & $(3,13)$ & 5.0 & $(3,11)$ & 4.0 & $(2,10)$ & 3. \\
\hline & & $(2,8)$ & 3.1 & $(1,7)$ & 2.3 & $(5,17)$ & 1.1 \\
\hline & & $(2,12)$ & 1.1 & & & & \\
\hline
\end{tabular}

TABLE III. Weights $\left|K_{a i N}\right|^{2}$ in $\%$ with $>1 \%$ contribution for the five electron system. The KS transition frequencies are $\Omega_{a i}=a-i$. Tabulated weights may not sum to $100 \%$ due to the absence of $<1 \%$ contributions. from the one-electron case. Is is exact in one-electron systems and should accurately predict the physics of asymptotic regions where the behaviour is essentially one-electron like. Scalar QCM is then conveniently expressed in (76), which provides a numerically simpler approximation to the full QCM, with some sacrifice of exact properties of the full theory in general systems. The scalar approximation could be used as a faster alternative to full QCM itself or as a doorway to further and cruder approximations. In particular it opens up analysis of transition frequencies and densities in a simple manner by relating information from the KS orbitals to the collective modes in the QCM.

Finally we test the approaches developed in this manuscript on a simple one-dimensional many-electron in Section VII. Results for this test system are generally excellent, demonstrating again the ability of the QCM to include the important physics of a many-electron system through the use of the displacement $\boldsymbol{u}$ only (or the equivalent $\boldsymbol{\xi}$ ).

Both reformulations presented here will aid in the development of a robust, basis set based, QCM approach for atomic and molecular systems. The difficulty in such approaches is the presence of non-constant denominators in $t_{\mu \nu}, \eta_{\alpha}$ and $S$. We believe, however, that a tractable means of dealing with these should be possible by using Gauss-Hermite quadrature with GTOs.

It should also be possible to further develop vdW functionals that use a further approximation to the QCM based on cleverly chosen orbital-like basis functions, possibly incorporating some unoccupied KS orbitals. Such functionals could incorporate ideas from Refs $15-17$ to be made even more efficient than the functional described here or in Ref. 7, without sacrificing the vital highfrequency, non-additive, and long-ranged physics that make the QCM attractive.

\section{ACKNOWLEDGMENTS}

We would like to thank Janos Angyan, Andreas Savin and Giovanni Vignale for much fruitful discussion. G. J. thanks John Dobson for his hospitality and support during a sabbatical stay in Brisbane. J.F.D. and T.G. were supported by ARC Discovery Grant DP1096240. I. V. T. was supported by the Spanish MICINN, Grant No. FIS2010-21282-C02-01, and "Grupos Consolidados UPV/EHU del Gobierno Vasco," Project No. IT-319-07.

\section{Appendix A: Kinetic stress tensor}

There is some debate over the appropriate form of the kinetic stress tensor $T^{\mathrm{Kin}}$. We have chosen the form given in (11) and (12), whereas the original QCM papers used the form given in (7). One requirement is that, for KohnSham systems, $\mathrm{T}^{0}$ obeys the force balance condition $\nabla$. $\mathrm{T}^{0}=-n^{0} \nabla V^{\mathrm{KS}}$ which is true both for the form (12) used 
in this manuscript and for equation 17 of Ref. 5 [leading to our equation (14) for a Kohn-Sham groundstate].

There are various ways to arrive at the possible forms of $T^{\mathrm{Kin}}(\boldsymbol{r})$ (see e.g. Refs 30-34). For completeness, we show one way to arrive at the form (11), (12) chosen for the present work. This form helps to make the QCM equations particularly simple. We base our form on the Wigner transform of the "classical" kinetic stress tensor. In a "classical" fluid with no current present, $T^{\mathrm{Kin}}$ is defined by

$$
T_{\mu \nu}^{\mathrm{Kin}}(\boldsymbol{r})=\int \mathrm{d} \boldsymbol{p} p_{\mu} p_{\nu} f(\boldsymbol{r}, \boldsymbol{p}) .
$$

Here $f$ is the classical one-body distribution function - i.e. the phase space probability density for finding a particle at position $\boldsymbol{r}$ with momentum $\boldsymbol{p}$. To model a quantum system we can replace $f(\boldsymbol{r}, \boldsymbol{p})$ by the Wigner form

$$
f(\boldsymbol{r}, \boldsymbol{p})=\int \frac{\mathrm{d} \boldsymbol{x}}{(2 \pi)^{3}} \rho\left(\boldsymbol{r}+\frac{\boldsymbol{x}}{2}, \boldsymbol{r}-\frac{\boldsymbol{x}}{2}\right) e^{i \boldsymbol{p} \cdot \boldsymbol{x}}
$$

where $\rho\left(\boldsymbol{r}, \boldsymbol{r}^{\prime}\right)$ is the quantal one-body density matrix. Using the short-hand $\rho=\rho(\boldsymbol{r}+\boldsymbol{x} / 2, \boldsymbol{r}-\boldsymbol{x} / 2)$ we thus find

$$
\begin{aligned}
T_{\mu \nu}^{\mathrm{Kin}}(\boldsymbol{r}) & =\int \mathrm{d} \boldsymbol{p} \int \frac{\mathrm{d} \boldsymbol{x}}{(2 \pi)^{3}} \rho p_{\mu} p_{\nu} e^{i \boldsymbol{p} \cdot \boldsymbol{x}} \\
& =\int \mathrm{d} \boldsymbol{p} \int \frac{\mathrm{d} \boldsymbol{x}}{(2 \pi)^{3}} \rho\left[-\partial_{x_{\mu}} \partial_{x_{\nu}} e^{i \boldsymbol{p} \cdot \boldsymbol{x}}\right] \\
& =\int \frac{\mathrm{d} \boldsymbol{x}}{(2 \pi)^{3}}\left[-\partial_{x_{\mu}} \partial_{x_{\nu}} \rho\right] \int \mathrm{d} \boldsymbol{p} e^{i \boldsymbol{p} \cdot \boldsymbol{x}} \\
& =\left[-\partial_{x_{\mu}} \partial_{x_{\nu}} \rho\right]_{\boldsymbol{x}=\mathbf{0}},
\end{aligned}
$$

and so, by the chain rule,

$$
\begin{aligned}
T_{\mu \nu}^{\mathrm{Kin}}(\boldsymbol{r}) & =-\left.\partial_{x_{\mu}} \partial_{x_{\nu}} \rho(\boldsymbol{r}+\boldsymbol{x} / 2, \boldsymbol{r}-\boldsymbol{x} / 2)\right|_{\boldsymbol{x}=\mathbf{0}} \\
& =\frac{1}{4}\left[\left(\partial_{\mu} \partial_{\nu}^{\prime}+\partial_{\mu}^{\prime} \partial_{\nu}-\partial_{\mu} \partial_{\nu}-\partial_{\mu}^{\prime} \partial_{\nu}^{\prime}\right) \rho\left(\boldsymbol{r}, \boldsymbol{r}^{\prime}\right)\right]_{\boldsymbol{r}=\boldsymbol{r}^{\prime}}
\end{aligned}
$$

with $\partial_{\mu} \equiv \partial_{r_{\mu}}$ and $\partial_{\mu}^{\prime} \equiv \partial_{r_{\mu}^{\prime}}$. Furthermore

$$
\begin{aligned}
& \frac{1}{4}\left[\left(\partial_{\mu} \partial_{\nu}+\partial_{\mu} \partial_{\nu}^{\prime}+\partial_{\mu}^{\prime} \partial_{\nu}+\partial_{\mu}^{\prime} \partial_{\nu}^{\prime}\right) \rho\left(\boldsymbol{r}, \boldsymbol{r}^{\prime}\right)\right]_{\boldsymbol{r}=\boldsymbol{r}^{\prime}} \\
& =\frac{1}{4} \partial_{\mu} \partial_{\nu} \rho(\boldsymbol{r}, \boldsymbol{r})=\frac{1}{4} \partial_{\mu} \partial_{\nu} n(\boldsymbol{r})
\end{aligned}
$$

Combining (A4) and (A5) we find

$$
\begin{aligned}
T_{\mu \nu}^{\operatorname{Kin}}(\boldsymbol{r})= & \frac{1}{2}\left[\left(\partial_{\mu} \partial_{\nu}^{\prime}+\partial_{\mu}^{\prime} \partial_{\nu}\right) \rho\left(\boldsymbol{r}, \boldsymbol{r}^{\prime}\right)\right]_{\boldsymbol{r}=\boldsymbol{r}^{\prime}} \\
& -\frac{1}{4} \partial_{\mu} \partial_{\nu} n(\boldsymbol{r}) .
\end{aligned}
$$

for a state with zero current, in agreement with (11).

Since $\rho\left(\boldsymbol{r}, \boldsymbol{r}^{\prime}\right)=\sum_{i} f_{i} \psi_{i}^{*}(\boldsymbol{r}) \psi_{i}\left(\boldsymbol{r}^{\prime}\right)$ in a groundstate KS system we find the stress tensor $\mathrm{T}^{0}$ for this situation to be

$$
\begin{aligned}
T_{\mu \nu}^{0}(\boldsymbol{r})= & \frac{1}{2} \sum_{i} f_{i}\left(\partial_{\mu} \partial_{\nu}^{\prime}+\partial_{\mu}^{\prime} \partial_{\nu}\right) \psi_{i}^{*}(\boldsymbol{r}) \psi_{i}\left(\boldsymbol{r}^{\prime}\right) \mid \boldsymbol{r}=\boldsymbol{r}^{\prime} \\
& -\frac{1}{4}\left[\partial_{\mu} \partial_{\nu} n^{0}(\boldsymbol{r})\right] \\
= & \Re \sum_{i} f_{i}\left[\partial_{\mu} \psi_{i}^{*}\right]\left[\partial_{\nu} \psi_{i}\right]-\frac{1}{4}\left[\partial_{\mu} \partial_{\nu} n^{0}\right]
\end{aligned}
$$

Thus (11) is exactly the Wigner version of the "classical" kinetic stress tensor (A1).

Using (13) we demonstrate that $\partial_{\alpha} T_{\alpha \mu}^{0}=-n^{0} V_{, \mu}^{\mathrm{KS}}$. Here we note that

$$
\partial_{\alpha} T_{\alpha \mu}^{0}=\frac{1}{2} \sum_{j} f_{j} t_{j \mu}
$$

where

$$
\begin{aligned}
t_{j \mu}= & \Re\left\{\left[\nabla^{2} \psi_{j}^{*}\right]\left[\partial_{\mu} \psi_{j}\right]+\left[\partial_{\alpha} \psi_{j}^{*}\right]\left[\partial_{\alpha} \partial_{\mu} \psi_{j}\right]\right. \\
& \left.-\left[\partial_{\alpha} \psi_{j}^{*}\right]\left[\partial_{\alpha} \partial_{\mu} \psi_{j}\right]-\psi_{j}^{*}\left[\partial_{\mu} \nabla^{2} \psi_{j}\right]\right\} \\
= & \Re\left\{\left[\nabla^{2} \psi_{j}^{*}\right]\left[\partial_{\mu} \psi_{j}\right]-\psi_{j}^{*}\left[\partial_{\mu} \nabla^{2} \psi_{j}\right]\right\} \\
= & -2 V_{, \mu}^{\mathrm{KS}}\left|\psi_{j}\right|^{2}
\end{aligned}
$$

and we have used the Schrödinger equation $\nabla^{2} \psi_{j}=$ $2\left(V^{\mathrm{KS}}-\epsilon_{j}\right) \psi_{j}$ to derive the final expression. Finally $\partial_{\alpha} T_{\mu \alpha}^{0}=-\sum_{j} f_{j} V_{, \mu}^{\mathrm{KS}}\left|\psi_{j}\right|^{2}=-n^{0} V_{, \mu}^{\mathrm{KS}}$ and the proof is complete.

Comparing this with the form $\overline{\mathrm{T}}^{0}(14)$ used in the original QCM formulation, we find $\partial_{\alpha} \bar{T}_{\mu \alpha}^{0}=\partial_{\alpha} T_{\mu \alpha}^{0}$ since $\partial_{\alpha}\left[\partial_{\mu} \partial_{\alpha} n^{0}\right]=\left[\partial_{\mu} \nabla^{2} n^{0}\right]=\partial_{\alpha} \delta_{\mu \alpha}\left[\nabla^{2} n^{0}\right]$. Since these are precisely the terms that differ between (12) and (14) it follows that the gradients must be identical.

\section{Appendix B: Compact form of the QCM equations}

In an earlier work ${ }^{7}$ we state without proof that equation (5) defined via (17) here (equations 2-5 of Ref. 7) are equivalent to equations 14-16 of Ref. 5. The only non-notational difference is in the kinetic force term $\boldsymbol{F}^{1 \mathrm{Kin}}=-\hat{\mathrm{K}} \boldsymbol{u}$ defined here via (15), in equation 14 of Ref. 5, and in equation 53 of Ref. 6 (abbreviated as G53) where it is derived from their equation C8 (abbreviated as GC8).

We demonstrate that the two forms are equivalent by working from GC8. While the same result can be obtained directly from G53 the derivation is less clear and less succinct. Specifically we must show that

$$
\boldsymbol{F}_{\mu}^{1 \mathrm{Kin}} \equiv \frac{\delta \mathcal{T}_{2}[\boldsymbol{u}]}{\delta u_{\mu}}=-\hat{K}_{\mu \nu} u_{\nu}
$$

where $\hat{K}_{\mu \nu}$ is defined in (15), since the remaining terms in (5) follow directly from equations 14-16 of Ref. 5. 
Following Gao et al ${ }^{6}$ we write GC8 as $\mathcal{T}_{2}[\boldsymbol{u}]=\int \mathrm{d} \boldsymbol{r} \mathcal{I}$ where

$$
\begin{aligned}
\mathcal{I}=\{ & \left\{\mathcal{K}_{\mu \nu}\left(4 U_{\mu \alpha} U_{\nu \alpha}-u_{\alpha, \mu} u_{\alpha, \nu}\right)+\frac{n^{0}}{8} U_{\alpha \alpha, \mu} U_{\nu \nu, \mu}\right. \\
& \left.+\frac{n_{, \nu}^{0}}{2} U_{\mu \nu} \partial_{\mu} U_{\alpha \alpha}+\frac{n_{, \nu}^{0}}{4} u_{\alpha, \mu} \partial_{\nu} u_{\mu, \alpha}\right\},
\end{aligned}
$$

with $U_{\mu \nu}=\frac{1}{2}\left(u_{\mu, \nu}+u_{\nu, \mu}\right), U_{\alpha \alpha}=u_{\alpha, \alpha}$, and $\mathcal{K}_{\mu \nu}=$ $\frac{1}{2} \Re \sum_{i} f_{i}\left[\partial_{\mu} \psi_{i}^{*}\right]\left[\partial_{\nu} \psi_{i}\right]$.

We can use integration by parts to remove derivatives of $n^{0}$ from the integrand $\mathcal{I}$. As such

$$
\begin{aligned}
\mathcal{T}_{2}[\boldsymbol{u}] & =\frac{1}{2} \int \mathrm{d} \boldsymbol{r}\left(2 \mathcal{K}_{\mu \nu} \mathcal{Y}_{\mu \nu}-\frac{n^{0}}{4} \mathcal{Z}\right) \\
& =\frac{1}{2} \int \mathrm{d} \boldsymbol{r}\left[T_{\mu \nu}^{0} \mathcal{Y}_{\mu \nu}+\frac{n^{0}}{4}\left(\mathcal{Y}_{\mu \nu, \mu \nu}-\mathcal{Z}\right)\right]
\end{aligned}
$$

where we define $T_{\mu \nu}^{0}=2 \mathcal{K}_{\mu \nu}-\frac{1}{4} n_{, \mu \nu}^{0}$ to be equal to (12) rather than the form appearing in Refs 5 and 6 . The undefined terms in the integrand of (B3) take the form

$$
\begin{aligned}
\mathcal{Y}_{\mu \nu}= & u_{\mu, \alpha} u_{\nu, \alpha}+u_{\alpha, \mu} u_{\nu, \alpha}+u_{\mu, \alpha} u_{\alpha, \nu} \\
\mathcal{Z}= & u_{\mu, \mu \alpha} u_{\nu, \nu \alpha}+2 u_{\mu, \nu \alpha} u_{\nu, \mu \alpha} \\
& +u_{\mu, \nu \alpha \alpha} u_{\nu, \mu}+u_{\mu, \nu} u_{\nu, \mu \alpha \alpha} \\
& +u_{\mu, \alpha \alpha} u_{\nu, \mu \nu}+u_{\mu, \nu \mu} u_{\nu, \alpha \alpha} \\
& +2 u_{\mu, \mu \nu \alpha} u_{\nu, \alpha}+2 u_{\mu, \alpha} u_{\nu, \mu \nu \alpha}
\end{aligned}
$$

where we have expanded $U_{\mu \nu}$ and used the product rule on all derivatives to arrive at these forms. The following identities are also used in the derivation of $\mathcal{Z}$ :

$$
\begin{aligned}
U_{\alpha \alpha, \mu} U_{\nu \nu, \mu} & =u_{\mu, \mu \alpha} u_{\nu, \nu \alpha} \\
2 \partial_{\nu} u_{\mu, \nu} \partial_{\mu} u_{\alpha, \alpha} & =\partial_{\alpha} u_{\mu, \alpha} \partial_{\mu} u_{\nu, \nu}+\partial_{\alpha} u_{\nu, \alpha} \partial_{\nu} u_{\mu, \mu}, \\
2 \partial_{\nu} u_{\nu, \mu} \partial_{\mu} u_{\alpha, \alpha} & =\partial_{\nu} u_{\nu, \alpha} \partial_{\alpha} u_{\mu, \mu}+\partial_{\mu} u_{\mu, \alpha} \partial_{\alpha} u_{\nu, \nu} \\
2 \partial_{\nu} u_{\alpha, \mu} \partial_{\nu} u_{\mu, \alpha} & =\partial_{\alpha} u_{\nu, \mu} \partial_{\alpha} u_{\mu, \nu}+\partial_{\alpha} u_{\mu, \nu} \partial_{\alpha} u_{\nu, \mu}
\end{aligned}
$$

which follow from exchange of indices under summation (eg. $A_{\nu \mu} B_{\mu \nu} \equiv A_{\mu \nu} B_{\nu \mu}$ ).

We can expand the terms of $\mathcal{Y}_{\mu \nu, \mu \nu}$ as follows:

$$
\begin{aligned}
\partial_{\mu} \partial_{\nu} u_{\mu, \alpha} u_{\nu, \alpha}= & u_{\mu, \mu \alpha} u_{\nu, \nu \alpha}+u_{\mu, \nu \alpha} u_{\nu, \mu \alpha} \\
& +u_{\mu, \mu \nu \alpha} u_{\nu, \alpha}+u_{\mu, \alpha} u_{\nu, \mu \nu \alpha} \\
\partial_{\mu} \partial_{\nu} u_{\mu, \alpha} u_{\alpha, \nu}= & u_{\mu, \mu \nu} u_{\nu, \alpha \alpha}+u_{\mu, \nu \alpha} u_{\nu, \mu \alpha} \\
& +u_{\mu, \mu \nu \alpha} u_{\nu, \alpha}+u_{\mu, \nu} u_{\nu, \mu \alpha \alpha} \\
\partial_{\mu} \partial_{\nu} u_{\nu, \alpha} u_{\alpha, \mu}= & u_{\mu, \alpha \alpha} u_{\nu, \mu \nu}+u_{\mu, \nu \alpha} u_{\nu, \mu \alpha} \\
& +u_{\mu, \alpha} u_{\nu, \mu \nu \alpha}+u_{\mu, \nu \alpha \alpha} u_{\nu, \mu}
\end{aligned}
$$

where we again exchange indices where appropriate. This leads to the following result

$$
\begin{aligned}
\mathcal{Y}_{\mu \nu, \mu \nu}= & u_{\mu, \mu \alpha} u_{\nu, \nu \alpha}+3 u_{\mu, \nu \alpha} u_{\nu, \mu \alpha} \\
& +u_{\mu, \alpha \alpha} u_{\nu, \mu \nu}+u_{\mu, \mu \nu} u_{\nu, \alpha \alpha} \\
& +u_{\mu, \nu \alpha \alpha} u_{\nu, \mu}+u_{\mu, \nu} u_{\nu, \mu \alpha \alpha} \\
& +2 u_{\mu, \alpha} u_{\nu, \mu \nu \alpha}+2 u_{\mu, \mu \nu \alpha} u_{\nu, \alpha} \\
= & \mathcal{Z}+u_{\mu, \nu \alpha} u_{\nu, \mu \alpha}
\end{aligned}
$$

and thus $\mathcal{Y}_{\mu \nu, \mu \nu}-\mathcal{Z}=u_{\nu, \mu \alpha} u_{\mu, \nu \alpha}$. The cancellation of so many terms is quite remarkable.

Finally (B3) becomes

$$
\begin{aligned}
\mathcal{T}_{2}[\boldsymbol{u}]= & \frac{1}{2} \int \mathrm{d} \boldsymbol{r}\left\{T_{\mu \nu}^{0}\left(u_{\mu, \alpha} u_{\nu, \alpha}+u_{\alpha, \mu} u_{\nu, \alpha}+u_{\mu, \alpha} u_{\alpha, \nu}\right)\right. \\
& \left.\quad+\left(n^{0} / 4\right) u_{\nu, \mu \alpha} u_{\mu, \nu \alpha}\right\} \\
= & -\frac{1}{2} \int \mathrm{d} \boldsymbol{r} u_{\mu} \hat{K}_{\mu \nu} u_{\nu} \\
\hat{K}_{\mu \nu}= & -\frac{1}{4} \partial_{\nu} \partial_{\alpha} n^{0} \partial_{\alpha} \partial_{\mu} \\
& +\partial_{\alpha} T_{\mu \nu}^{0} \partial_{\alpha}+\partial_{\alpha} T_{\alpha \nu}^{0} \partial_{\mu}+\partial_{\nu} T_{\alpha \mu}^{0} \partial_{\alpha} .
\end{aligned}
$$

Here we used integration by parts on the derivatives of $u_{\mu}$ to obtain (B9) and (B10). The operator $\hat{\mathrm{K}}$ defined in (B10) is identical to that defined in (15). Taking the functional derivative w.r.t. $u_{\mu}$ thus gives

$$
\boldsymbol{F}_{\mu}^{1 \mathrm{Kin}}=\frac{\delta \mathcal{T}_{2}[\boldsymbol{u}]}{\delta u_{\mu}}=-\hat{K}_{\mu \nu} u_{\nu}
$$

and it is clear that (B1) is satisfied.

\section{Appendix C: Correlation energy expressions}

Let us first define the projected Coulomb operator $W_{N M}=\left\langle\boldsymbol{u}_{M}|\hat{\mathbf{Q}}| \boldsymbol{u}_{N}\right\rangle$ where $\hat{\mathbf{Q}}$ is defined in (19) or (20). This can be written as

$$
\begin{aligned}
W_{N M} & =\int \mathrm{d} \boldsymbol{r} \boldsymbol{u}_{M}^{*}(\boldsymbol{r}) \cdot\left[\hat{\mathrm{Q}} \boldsymbol{u}_{N}\right](\boldsymbol{r}) \\
& =\int \mathrm{d} \boldsymbol{r} \mathrm{d} \boldsymbol{r}^{\prime} \boldsymbol{u}_{M}^{*}(\boldsymbol{r}) \cdot \mathrm{Q}\left(\boldsymbol{r}, \boldsymbol{r}^{\prime}\right) \cdot \boldsymbol{u}_{N}\left(\boldsymbol{r}^{\prime}\right) \\
& =\int \frac{\mathrm{d} \boldsymbol{r} \mathrm{d} \boldsymbol{r}^{\prime}}{\left|\boldsymbol{r}-\boldsymbol{r}^{\prime}\right|} d_{M}^{*}(\boldsymbol{r}) d_{N}\left(\boldsymbol{r}^{\prime}\right) .
\end{aligned}
$$

where similar equivalences hold true for $W_{N M \lambda}=$ $\left\langle\boldsymbol{u}_{M \lambda}|\hat{\mathrm{Q}}| \boldsymbol{u}_{N \lambda}\right\rangle$ or for alternative forms of the Coulomb potential (e.g. range-separated).

Using equations (21)-(22) and (27)-(28) we can write the correlation energy as

$$
\begin{aligned}
E_{\mathrm{c}}= & \frac{1}{2} \int_{0}^{1} \mathrm{~d} \lambda \int_{0}^{\infty} \frac{\mathrm{d} \sigma}{\pi} \int \frac{\mathrm{d} \boldsymbol{r} \mathrm{d} \boldsymbol{r}^{\prime}}{\left|\boldsymbol{r}-\boldsymbol{r}^{\prime}\right|} \\
& \times \sum_{N}\left(\frac{d_{N \lambda}^{*}(\boldsymbol{r}) d_{N \lambda}\left(\boldsymbol{r}^{\prime}\right)}{\Omega_{N \lambda}^{2}+\sigma^{2}}-\frac{d_{N}^{*}(\boldsymbol{r}) d_{N}\left(\boldsymbol{r}^{\prime}\right)}{\Omega_{N}^{2}+\sigma^{2}}\right) \\
= & \frac{1}{2} \int_{0}^{1} \mathrm{~d} \lambda \int_{0}^{\infty} \frac{\mathrm{d} \sigma}{\pi} \sum_{N}\left(\frac{W_{N N \lambda}}{\Omega_{N \lambda}^{2}+\sigma^{2}}-\frac{W_{N N}}{\Omega_{N}^{2}+\sigma^{2}}\right) \\
= & \frac{1}{2} \int_{0}^{1} \mathrm{~d} \lambda \sum_{N}\left(\frac{W_{N N \lambda}}{2 \Omega_{N \lambda}}-\frac{W_{N N}}{2 \Omega_{N}}\right) .
\end{aligned}
$$

Here the governing eigen-equations for $\boldsymbol{u}_{N \lambda}$ are as defined in (22)

$$
\Omega_{N \lambda}^{2} n^{0} \boldsymbol{u}_{N \lambda}=(\hat{\mathrm{R}}+\lambda \hat{\mathbf{Q}}) \boldsymbol{u}_{N \lambda}
$$


with normalisation $\int n^{0}(\boldsymbol{r}) \boldsymbol{u}_{N \lambda}^{*}(\boldsymbol{r}) \cdot \boldsymbol{u}_{M \lambda}(\boldsymbol{r})=\delta_{N M}$.

Following the ideas of Furche, ${ }^{26}$ we can take the $\lambda$ derivative of $\int \mathrm{d} \boldsymbol{r} \boldsymbol{u}_{N \lambda} \cdot(\mathrm{C} 7)$ to work directly from $(\mathrm{C} 6)$. Here

$$
2 \Omega_{N \lambda}\left[\partial_{\lambda} \Omega_{N \lambda}\right]=\int \mathrm{d} \boldsymbol{r} \boldsymbol{u}_{N \lambda}^{*} \cdot \hat{\mathbf{Q}} \boldsymbol{u}_{N \lambda}
$$

where derivatives of $\boldsymbol{u}_{N \lambda}$ can be ignored by the HellmanFeynman theorem. Thus

$$
\left[\partial_{\lambda} \Omega_{N \lambda}\right]=\int \mathrm{d} \boldsymbol{r} \frac{\boldsymbol{u}_{N \lambda}^{*}(\boldsymbol{r})\left[\hat{\mathbf{Q}} \boldsymbol{u}_{N \lambda}\right](\boldsymbol{r})}{2 \Omega_{N \lambda}}=\frac{W_{N N \lambda}}{2 \Omega_{N \lambda}} .
$$

and we can write the correlation as a sum over zero-point energies such that

$$
\begin{aligned}
E_{\mathrm{c}} & =\frac{1}{2} \int_{0}^{1} \mathrm{~d} \lambda \sum_{N}\left(\left[\partial_{\lambda} \Omega_{N \lambda}\right]-\frac{W_{N N}}{2 \Omega_{N}}\right) \\
& =\frac{1}{2} \sum_{N}\left[\bar{\Omega}_{N}-\Omega_{N}\left(1+\frac{W_{N N}}{2 \Omega_{N}^{2}}\right)\right]
\end{aligned}
$$

where $\bar{\Omega}_{N}=\Omega_{N 1}$. In certain systems it may make sense to work with exchange and correlation together. Here

$$
E_{\mathrm{xc}}=\frac{1}{2} \sum_{N}\left[\bar{\Omega}_{N}-\Omega_{N}\right]-\frac{1}{2} \int \mathrm{d} \boldsymbol{r} n^{0}(\boldsymbol{r}) w_{C}(\boldsymbol{r})
$$

where $w_{C}(\boldsymbol{r})=\int \mathrm{d} \boldsymbol{r}^{\prime} \delta\left(\boldsymbol{r}-\boldsymbol{r}^{\prime}\right) V_{C}\left(\left|\boldsymbol{r}-\boldsymbol{r}^{\prime}\right|\right)$ is like the Coulomb potential at zero distance and is ill-defined for a true Coulomb potential $V_{C}(R)=1 / R$. However it becomes well-defined if we replace the Coulomb potential by a range-separated ${ }^{35-37}$ form eg. $V_{C}^{\left(q_{\mathrm{RS}}\right)}(R)=$ $\operatorname{erf}\left(q_{\mathrm{RS}} R\right) / R$.

To solve for $\bar{\Omega}_{N}$ involves a difficult diagonalisation of $\hat{\mathrm{R}}+\hat{\mathrm{Q}}$ and may be best avoided. We can use the complete and orthogonal nature of $\boldsymbol{u}_{N \lambda}$ and $\boldsymbol{u}_{N}$ to write $\boldsymbol{u}_{N \lambda}=$ $\sum_{K} U_{N K} \boldsymbol{u}_{K}$ where $\mathbb{U}^{\dagger} \mathbb{U}=\mathbb{I}$. Thus we can write

$$
\chi_{\lambda}\left(\boldsymbol{r}, \boldsymbol{r}^{\prime}\right)=-\Re \sum_{N M} X_{N M \lambda} d_{N}^{*}(\boldsymbol{r}) d_{M}\left(\boldsymbol{r}^{\prime}\right)
$$

where $X_{N M 0}=\delta_{N M} /\left(\sigma^{2}+\Omega_{N}^{2}\right)$ or $\mathbb{X}_{0}=\left(\sigma^{2}+\mathbb{L}\right)^{-1}$ where $L_{N M}=\delta_{N M} \Omega_{N}^{2}$. Solving for $\hat{\chi}_{\lambda}=\hat{\chi}_{0}+\lambda \hat{\chi}_{0} \hat{v} \hat{\chi}_{\lambda}$ gives

$$
\mathbb{X}_{\lambda}=\mathbb{X}_{0}-\lambda \mathbb{X}_{0} \mathbb{W}_{\lambda}, \quad \mathbb{X}_{\lambda}=\frac{\mathbb{I}}{\sigma^{2}+\mathbb{L}+\lambda \mathbb{W}}
$$

This corresponds to

$$
\begin{aligned}
E_{\mathrm{c}}= & \frac{1}{2} \int_{0}^{1} \mathrm{~d} \lambda \int_{0}^{\infty} \frac{\mathrm{d} \sigma}{\pi} \\
& \times \operatorname{Tr}\left[\frac{\mathbb{W}}{\sigma^{2}+\mathbb{L}+\lambda \mathbb{W}}-\frac{\mathbb{W}}{\sigma^{2}+\mathbb{L}}\right]
\end{aligned}
$$

which can sometimes prove useful in real calculations
We can relate all this back to (C11). Solving the eigenequation $\mathbb{V D}_{\lambda}=(\mathbb{L}+\lambda \mathbb{W}) \mathbb{V}$ gives

$$
\begin{aligned}
X_{M N \lambda} & =\left[\mathbb{V}\left(\sigma^{2}+\mathbb{D}_{\lambda}\right)^{-1} \mathbb{V}^{\dagger}\right]_{M N} \\
& =\sum_{K} U_{K M}^{*}\left(\sigma^{2}+\Omega_{K \lambda}^{2}\right)^{-1} U_{K N} .
\end{aligned}
$$

With appropriate sorting of the eigen-value/vector pairs it is clear that the eigenvalues $D_{N N 1}$ of $\mathbb{L}+\mathbb{W}$ are $\bar{\Omega}_{N}^{2}=\Omega_{N 1}^{2}$ and $\mathbb{U}=\mathbb{V}^{\dagger}$. Thus the two expressions are equivalent.

Furthermore for $\Omega_{N}^{2} \gg \bar{W}_{N}$ where $\bar{W}_{N}=\sum_{M}\left|W_{N M}\right|$ we can solve the eigen-problem perturbatively so that $\bar{\Omega}_{N}^{2} \approx \Omega_{N}^{2}+W_{N N}$. Thus $\bar{\Omega}_{N}=\sqrt{\Omega_{N}^{2}+W_{N N}} \approx \Omega_{N}(1+$ $\left.\frac{W_{N N}}{2 \Omega_{N}^{2}}-\frac{W_{N N}^{2}}{8 \Omega_{N}^{4}}\right)$. We can speed calculation and improve numerical stability by choosing an $N^{*}$ above which we use the approximation. Setting $\beta_{N}=\frac{W_{N N}}{2 \Omega_{N}^{2}}$ we then find

$$
E_{\mathrm{c}} \approx \frac{1}{2} \sum_{N=0}^{N^{*}}\left[\bar{\Omega}_{N}-\Omega_{N}\left(1+\beta_{N}\right)\right]-\sum_{N>N^{*}} \frac{\Omega_{N} \beta_{N}^{2}}{4}
$$

Such a perturbative approach will be almost guaranteed convergent, and with a fairly small $\Omega_{N^{*}}$ if rangeseparation is used.

\section{Appendix D: One-Electron governing operator}

As discussed in Section V [equations (61)-(67)] we can obtain the exact linear perturbation solutions in a oneelectron system via a solution of

$$
\omega^{2} \boldsymbol{\xi}=\hat{\mathrm{R}}^{1 e^{-}} \boldsymbol{\xi}
$$

where the associated change in density is $n^{1}=-\nabla$. $\psi \boldsymbol{\xi}^{1 e^{-}}$. Since this is an identical problem to finding the QCM solutions via

$$
\omega^{2} \boldsymbol{\xi}=\hat{\mathrm{R}}^{(\boldsymbol{\xi})} \boldsymbol{\xi}
$$

it is clear that if $\hat{\mathrm{R}}^{1 e^{-}}=\hat{\mathrm{R}}^{(\boldsymbol{\xi})}$ then the QCM is exact for one-electron systems.

For a one-electron system we set $V=V^{\mathrm{KS}}-\epsilon_{0}$ such that the groundstate occupied orbital $\psi$ is a solution of $\left[-\frac{1}{2} \nabla^{2}+V\right] \psi=0$. Equations (55) thus become $\eta_{\alpha}=$ $\partial_{\alpha} \log |\psi|$ and $S=2 V$ and thus $\hat{h}=-\frac{1}{2}\left(\nabla^{2}-S\right)$. The former can be used to derive the following

$$
\begin{aligned}
{\left[\partial_{\mu} \eta_{\nu}\right] } & =\left[\partial_{\nu} \eta_{\mu}\right]=-2 t_{\mu \nu} \\
{\left[\nabla^{2} \eta_{\mu}\right] } & =-2\left[\partial_{\alpha} t_{\alpha \mu}\right]=S_{, \mu}+4 \eta_{\alpha} t_{\alpha \mu}
\end{aligned}
$$

where these identities will be used throughout this appendix.

Comparing the final term of (67) with (17) we wish to swap $\left(\partial_{\mu}-\eta_{\mu}\right)$ and $\left(\partial_{\nu}+\eta_{\nu}\right)$ across sides. This can be 
done by repeatedly using

$$
\begin{aligned}
\partial_{\mu} f & =f \partial_{\mu}+\left[\partial_{\mu} f\right] \\
\nabla^{2} f & =f \nabla^{2}+\left[\nabla^{2} f\right]+2\left[\partial_{\alpha} f\right] \partial_{\alpha} \\
& =f \nabla^{2}-\left[\nabla^{2} f\right]+2 \partial_{\alpha}\left[\partial_{\alpha} f\right] \\
f \nabla^{2} & =\nabla^{2} f+\left[\nabla^{2} f\right]-2 \partial_{\alpha}\left[\partial_{\alpha} f\right] \\
& =\nabla^{2} f-\left[\nabla^{2} f\right]-2\left[\partial_{\alpha} f\right] \partial_{\alpha}
\end{aligned}
$$

in the appropriate places.

We first address the terms involving $S$. These are

$$
\begin{aligned}
\partial_{\mu} S \partial_{\nu} & =\partial_{\nu} S \partial_{\mu}+S_{, \mu} \partial_{\nu}-\partial_{\mu} S_{, \nu}+S_{, \mu \nu} \\
\partial_{\mu} S \eta_{\nu} & =\eta_{\nu} S \partial_{\mu}+\left[\partial_{\mu} \eta_{\nu} S\right] \\
-\eta_{\mu} S \partial_{\nu} & =-\partial_{\nu} S \eta_{\nu}+\left[\partial_{\nu} \eta_{\mu} S\right]
\end{aligned}
$$

and thus

$$
\begin{aligned}
\hat{R}_{\mu \nu-S}^{1 e^{-}}= & \left(\partial_{\mu}-\eta_{\mu}\right) S\left(\partial_{\nu}+\eta_{\nu}\right) \\
= & \left(\partial_{\nu}+\eta_{\nu}\right) S\left(\partial_{\mu}-\eta_{\mu}\right) \\
& +S_{, \mu} \partial_{\nu}-\partial_{\mu} S_{, \nu}+S_{, \mu \nu} \\
& +\left[\partial_{\mu} \eta_{\nu} S\right]+\left[\partial_{\nu} \eta_{\mu} S\right] .
\end{aligned}
$$

The terms involving the Laplacian are a little more difficult to deal with. Here

$$
\begin{aligned}
\partial_{\mu} \nabla^{2} \eta_{\nu}= & \eta_{\nu} \nabla^{2} \partial_{\mu}-2 \nabla^{2} t_{\mu \nu} \\
& -S_{, \nu} \partial_{\mu}+4 \eta_{\alpha} t_{\alpha \nu} \partial_{\mu}-4 \partial_{\alpha} t_{\alpha \nu} \partial_{\mu} \\
-\eta_{\mu} \nabla^{2} \partial_{\nu}= & -\partial_{\nu} \nabla^{2} \eta_{\mu}-2 t_{\mu \nu} \nabla^{2} \\
& +\partial_{\nu} S_{, \mu}+4 \partial_{\nu} t_{\alpha \mu} \eta_{\alpha}-4 \partial_{\nu} t_{\alpha \mu} \partial_{\alpha}
\end{aligned}
$$

and

$$
\begin{aligned}
\eta_{\mu} \nabla^{2} \eta_{\nu}= & \left(\partial_{\alpha} \eta_{\mu}-\left[\partial_{\alpha} \eta_{\mu}\right]\right)\left(\eta_{\nu} \partial_{\alpha}+\left[\partial_{\alpha} \eta_{\nu}\right]\right) \\
= & \left(\eta_{\nu} \partial_{\alpha}+\left[\partial_{\alpha} \eta_{\nu}\right]\right)\left(\partial_{\alpha} \eta_{\mu}-\left[\partial_{\alpha} \eta_{\mu}\right]\right) \\
& -\left[\partial_{\alpha} \eta_{\mu}\right] \eta_{\nu} \partial_{\alpha}+\partial_{\alpha} \eta_{\mu}\left[\partial_{\alpha} \eta_{\nu}\right]-\left[\partial_{\alpha} \eta_{\mu}\right]\left[\partial_{\alpha} \eta_{\nu}\right] \\
= & \eta_{\nu} \nabla^{2} \eta_{\mu}-2\left[\partial_{\alpha} \eta_{\nu}\right]\left[\partial_{\alpha} \eta_{\mu}\right] \\
& -\eta_{\nu}\left[\nabla^{2} \eta_{\mu}\right]-\left[\nabla^{2} \eta_{\nu}\right] \eta_{\mu} \\
& -2 \eta_{\nu}\left[\partial_{\alpha} \eta_{\mu}\right] \partial_{\alpha}+2 \partial_{\alpha}\left[\partial_{\alpha} \eta_{\nu}\right] \eta_{\mu} \\
= & \eta_{\nu} \nabla^{2} \eta_{\mu}-2\left[\partial_{\alpha} \eta_{\nu}\right]\left[\partial_{\alpha} \eta_{\mu}\right] \\
& -\eta_{\nu} S_{, \mu}-\eta_{\mu} S_{, \nu}-4 \eta_{\nu} t_{\alpha \mu} \eta_{\alpha}-4 \eta_{\alpha} t_{\alpha \nu} \eta_{\mu} \\
& +4 \eta_{\nu} t_{\alpha \mu} \partial_{\alpha}-4 \partial_{\alpha} t_{\alpha \nu} \eta_{\mu} .
\end{aligned}
$$

Combining the above lets us write

$$
\begin{aligned}
\hat{R}_{\mu \nu-L}^{1 e^{-}}= & \left(\partial_{\mu}-\eta_{\mu}\right) \nabla^{2}\left(\partial_{\nu}+\eta_{\nu}\right) \\
= & \left(\partial_{\nu}+\eta_{\nu}\right) \nabla^{2}\left(\partial_{\mu}-\eta_{\mu}\right)-4 \partial_{\alpha} t_{\mu \nu} \partial_{\alpha} \\
& -4\left(\partial_{\alpha}+\eta_{\alpha}\right) t_{\alpha \nu}\left(\partial_{\mu}-\eta_{\mu}\right) \\
& -4\left(\partial_{\nu}+\eta_{\nu}\right) t_{\alpha \mu}\left(\partial_{\alpha}-\eta_{\alpha}\right) \\
& +2\left[\partial_{\alpha} \eta_{\nu}\right]\left[\partial_{\alpha} \eta_{\mu}\right]-2\left[\nabla^{2} t_{\mu \nu}\right] \\
& +\partial_{\nu} S_{, \mu}-S_{, \nu} \partial_{\mu}+\eta_{\nu} S_{, \mu}+\eta_{\mu} S_{, \nu}
\end{aligned}
$$

We can now use (D6) and (D4) together to show

$$
\begin{aligned}
4 \hat{R}_{\mu \nu}^{1 e^{-}}= & \hat{R}_{\mu \nu-L}^{1 e^{-}}-\hat{R}_{\mu \nu-S}^{1 e^{-}} \\
= & \left(\partial_{\nu}+\eta_{\nu}\right)\left(\nabla^{2}-S\right)\left(\partial_{\mu}-\eta_{\mu}\right) \\
& -4\left(\partial_{\alpha}+\eta_{\alpha}\right) t_{\mu \nu}\left(\partial_{\alpha}-\eta_{\alpha}\right) \\
& -4\left(\partial_{\alpha}+\eta_{\alpha}\right) t_{\alpha \nu}\left(\partial_{\mu}-\eta_{\mu}\right) \\
& -4\left(\partial_{\nu}+\eta_{\nu}\right) t_{\alpha \mu}\left(\partial_{\alpha}-\eta_{\alpha}\right) \\
& +S_{, \mu \nu}+2\left[\partial_{\alpha} \eta_{\nu}\right]\left[\partial_{\alpha} \eta_{\mu}\right] \\
& -2\left[\nabla^{2} t_{\mu \nu}\right]-4\left[\eta_{\alpha} \partial_{\alpha} t_{\mu \nu}\right]
\end{aligned}
$$

where we cancel most terms via

$$
\begin{aligned}
\partial_{\alpha} t_{\mu \nu} \partial_{\alpha}= & \left(\partial_{\alpha}+\eta_{\alpha}\right) t_{\mu \nu}\left(\partial_{\alpha}-\eta_{\alpha}\right) \\
& +\left[\eta_{\alpha} \partial_{\alpha} t_{\mu \nu}\right]+S t_{\mu \nu} \\
{\left[\partial_{\mu} \eta_{\nu} S\right]=} & -2 S t_{\mu \nu}+\left[\eta_{\nu} S_{, \mu}\right] \\
{\left[\partial_{\nu} \eta_{\mu} S\right]=} & -2 S t_{\mu \nu}+\left[\eta_{\mu} S_{, \nu}\right] .
\end{aligned}
$$

The operator terms are now the same as those of (50). The remaining constant is

$$
\begin{aligned}
4 K_{\mu \nu}= & S_{, \mu \nu}+2\left[\partial_{\alpha} \eta_{\nu}\right]\left[\partial_{\alpha} \eta_{\mu}\right]-2\left[\nabla^{2} t_{\mu \nu}\right]-4\left[\eta_{\alpha} \partial_{\alpha} t_{\mu \nu}\right] \\
= & S_{, \mu \nu}+2\left[\partial_{\alpha} \eta_{\nu}\right]\left[\partial_{\alpha} \eta_{\mu}\right]+S_{, \mu \nu} \\
& -2\left[\partial_{\nu} \eta_{\alpha} \partial_{\mu} \eta_{\alpha}\right]+2\left[\eta_{\alpha} \partial_{\alpha} \partial_{\mu} \eta_{\nu}\right] \\
= & 2 S_{, \mu \nu}=4 V_{, \mu \nu}
\end{aligned}
$$

where we have used $\left[\nabla^{2} t_{\mu \nu}\right]=-\frac{1}{2} S_{, \mu \nu}+\left[\partial_{\nu} \eta_{\alpha}\right]\left[\partial_{\mu} \eta_{\alpha}\right]+$ $\left[\eta_{\alpha} \partial_{\nu} \partial_{\mu} \eta_{\alpha}\right]$ and $\left[\partial_{\alpha} \eta_{\nu}\right]=\left[\partial_{\nu} \eta_{\alpha}\right]$ to arrive at the final form.

Thus (D8) becomes

$$
\begin{aligned}
\hat{R}_{\mu \nu}^{1 e^{-}=} & V_{, \mu \nu}+\frac{1}{4}\left(\partial_{\nu}+\eta_{\nu}\right)\left(\nabla^{2}-S\right)\left(\partial_{\mu}-\eta_{\mu}\right) \\
& -\left(\partial_{\alpha}+\eta_{\alpha}\right) t_{\mu \nu}\left(\partial_{\alpha}-\eta_{\alpha}\right) \\
& -\left(\partial_{\alpha}+\eta_{\alpha}\right) t_{\alpha \nu}\left(\partial_{\mu}-\eta_{\mu}\right) \\
& -\left(\partial_{\nu}+\eta_{\nu}\right) t_{\alpha \mu}\left(\partial_{\alpha}-\eta_{\alpha}\right)
\end{aligned}
$$

which is identical to $\hat{R}_{\mu \nu}^{(\boldsymbol{\xi})}$ and through it $\hat{R}_{\mu \nu}$ via (45).

Finally, since $\psi^{1}$ and through it $\phi^{1}$ and $n^{1}$ can be calcuted from $\boldsymbol{\xi}$ it is clear that a solution to the QCM is identical to a linearised solution of the Schrödinger equation for a one-electron systen, and vice versa. 


\section{Appendix E: Scalar QCM operator in the KS basis}

Inserting (50) [and setting $\left(\nabla^{2}-S\right)=\left(\partial_{\alpha}+\eta_{\alpha}\right)\left(\partial_{\alpha}-\right.$ $\left.\eta_{\alpha}\right)$ ] into (85) and using integration by parts we find

$$
\begin{aligned}
R_{j k}= & \int \mathrm{d} \boldsymbol{r}\left[\hat{D}_{\mu} \psi_{j}^{*}\right] V_{, \mu \nu}\left[\hat{D}_{\nu} \psi_{k}\right] \\
& +\frac{1}{4} \int \mathrm{d} \boldsymbol{r}\left[\hat{D}_{\alpha} \hat{D}_{\mu} \hat{D}_{\nu} \psi_{j}^{*}\right]\left[\hat{D}_{\alpha} \hat{D}_{\nu} \hat{D}_{\mu} \psi_{k}\right] \\
& +\int \mathrm{d} \boldsymbol{r}\left[\hat{D}_{\alpha} \hat{D}_{\mu} \psi_{j}^{*}\right] t_{\mu \nu}\left[\hat{D}_{\alpha} \hat{D}_{\nu} \psi_{k}\right] \\
& +\int \mathrm{d} \boldsymbol{r}\left[\hat{D}_{\alpha} \hat{D}_{\mu} \psi_{j}^{*}\right] t_{\alpha \nu}\left[\hat{D}_{\mu} \hat{D}_{\nu} \psi_{k}\right] \\
& +\int \mathrm{d} \boldsymbol{r}\left[\hat{D}_{\nu} \hat{D}_{\mu} \psi_{j}^{*}\right] t_{\alpha \mu}\left[\hat{D}_{\alpha} \hat{D}_{\nu} \psi_{k}\right]
\end{aligned}
$$

where we have used the shorthand $\hat{D}_{\alpha}=\partial_{\alpha}-\eta_{\alpha}$.

It follows from $\boldsymbol{\eta}=\frac{1}{2} \nabla \log n^{0}$ that $\left[\partial_{\mu} \eta_{\nu}\right]=\left[\partial_{\nu} \eta_{\mu}\right]$ and thus $\partial_{\mu} \eta_{\nu}+\eta_{\mu} \partial_{\nu}=\eta_{\nu} \partial_{\mu}+\partial_{\nu} \eta_{\mu}$. Therefore $\hat{D}_{\mu} \hat{D}_{\nu}=$ $\hat{D}_{\nu} \hat{D}_{\mu}$ and we can simplify (E1) (noting that we can also swap Greek indices as they are summed over) to

$$
\begin{aligned}
R_{j k}= & \int \mathrm{d} \boldsymbol{r}\left[\hat{D}_{\mu} \psi_{j}^{*}\right] V_{, \mu \nu}\left[\hat{D}_{\nu} \psi_{k}\right] \\
& +\frac{1}{4} \int \mathrm{d} \boldsymbol{r}\left[\hat{D}_{\alpha} \hat{D}_{\mu} \hat{D}_{\nu} \psi_{j}^{*}\right]\left[\hat{D}_{\alpha} \hat{D}_{\mu} \hat{D}_{\nu} \psi_{k}\right] \\
& +3 \int \mathrm{d} \boldsymbol{r}\left[\hat{D}_{\alpha} \hat{D}_{\mu} \psi_{j}^{*}\right] t_{\mu \nu}\left[\hat{D}_{\alpha} \hat{D}_{\nu} \psi_{k}\right] .
\end{aligned}
$$

\section{REFERENCES}

${ }^{1}$ E. Madelung, Naturwissenschaften 14, 1004 (1926); Zeitschrift für Physik A Hadrons and Nuclei 40, 322 (1927).

${ }^{2}$ H.-V. Nguyen and S. de Gironcoli, Phys. Rev. B 79, 205114 (2009).

${ }^{3}$ I. V. Tokatly, Phys. Rev. B 71, 165104 (2005); 71, 165105 (2005).

${ }^{4}$ I. V. Tokatly, Phys. Rev. B 75, 125105 (2007).

${ }^{5}$ J. Tao, X. Gao, G. Vignale, and I. V. Tokatly, Phys. Rev. Lett. 103, 086401 (2009).

${ }^{6}$ X. Gao, J. Tao, G. Vignale, and I. V. Tokatly, Phys. Rev. B 81, 195106 (2010).

${ }^{7}$ T. Gould and J. F. Dobson, Phys. Rev. B 84, 241108 (2011).

${ }^{8}$ P. Hohenberg and W. Kohn, Phys. Rev. 136, B864 (1964); W. Kohn and L. J. Sham, 140 (1965).

${ }^{9}$ E. Runge and E. K. U. Gross, Phys. Rev. Lett. 52, 997 (1984).

${ }^{10}$ See page 1898 of R. H. French, V. A. Parsegian, R. Podgornik, R. F. Rajter, A. Jagota, J. Luo, D. Asthagiri, M. K. Chaudhury,
Y.-m. Chiang, S. Granick, S. Kalinin, M. Kardar, R. Kjellander, D. C. Langreth, J. Lewis, S. Lustig, D. Wesolowski, J. S. Wettlaufer, W.-Y. Ching, M. Finnis, F. Houlihan, O. A. von Lilienfeld, C. J. van Oss, and T. Zemb, Rev. Mod. Phys. 82, 1887 (2010).

${ }^{11}$ J. Harl and G. Kresse, Phys. Rev. B 77, 045136 (2008); Phys. Rev. Lett. 103, 056401 (2009).

${ }^{12}$ S. Lebègue, J. Harl, T. Gould, J. G. Ángyán, G. Kresse, and J. F. Dobson, Phys. Rev. Lett. 105, 196401 (2010).

${ }^{13} \mathrm{H}$. Eshuis, J. Yarkony, and F. Furche, J. Chem. Phys. 132, 234114 (2010); H. Eshuis and F. Furche, J. Phys. Chem. Lett. 2, 983 (2011); H. Eshuis, J. Bates, and F. Furche, Theoretical Chemistry Accounts: Theory, Computation, and Modeling (Theoretica Chimica Acta) 131, 1 (2012), 10.1007/s00214-0111084-8.

${ }^{14}$ J. P. Perdew and K. Schmidt, "Density functional theory and its application to materials," (Springer, 2001); J. P. Perdew, A. Ruzsinszky, J. Tao, V. N. Staroverov, G. E. Scuseria, and G. I. Csonka, J. Chem. Phys. 123, 062201 (2005).

${ }^{15}$ S. Grimme, J. Comp. Chem. 27, 1787 (2006).

${ }^{16}$ A. Tkatchenko and M. Scheffler, Phys. Rev. Lett. 102, 073005 (2009).

${ }^{17}$ O. A. Vydrov and T. Van Voorhis, Phys. Rev. Lett. 103, 063004 (2009).

${ }^{18}$ M. Dion, H. Rydberg, E. Schröder, D. C. Langreth, and B. I. Lundqvist, Phys. Rev. Lett. 92, 246401 (2004).

${ }^{19}$ H. Rydberg, B. I. Lundqvist, D. C. Langreth, and M. Dion, Phys. Rev. B 62, 6997 (2000).

${ }^{20}$ H. Rydberg, M. Dion, N. Jacobson, E. Schröder, P. Hyldgaard, S. I. Simak, D. C. Langreth, and B. I. Lundqvist, Phys. Rev. Let. 91, 126402 (2003).

${ }^{21}$ D. C. Langreth, M. Dion, H. Rydberg, E. Schrder, P. Hyldgaard, and B. I. Lundqvist, Int. J. Quantum Chem. 101, 599 (2005).

${ }^{22}$ J. F. Dobson and T. Gould, Journal of Physics: Condensed Matter 24, 073201 (2012).

${ }^{23}$ W. J. Hehre, R. F. Stewart, and J. A. Pople, J. Chem. Phys. 51, 2657 (1969); M. D. Newton, 51, 3917 (1969).

${ }^{24}$ J. F. Dobson, Phys. Rev. Lett. 73, 2244 (1994).

${ }^{25} \mathrm{~S}$. Pittalis, G. Vignale, and I. V. Tokatly, Phys. Rev. B 84, 245118 (2011).

${ }^{26}$ F. Furche, J. Chem. Phys. 129, 114105 (2008).

${ }^{27}$ T. C. Wallstrom, Phys. Rev. A 49, 1613 (1994).

${ }^{28}$ For one-electron this is true by construction. In the $1 \mathrm{D}$ cases it follows from the fact that in 1D a scalar function can be written as the derivative of another function without the curl.

${ }^{29}$ J. F. Dobson, J. Chem. Phys. 94, 4328 (1991); 98, 8870 (1993).

${ }^{30}$ E. Schrödinger, Ann. Phys. (Leipzig) 82, 265 (1927).

${ }^{31} \mathrm{~W}$. Pauli, Handbuch der Physik, edited by H. Geiger and K. Scheel (Springer, Berlin, 1933).

${ }^{32}$ R. P. Feynman, Forces and Stresses in Molecules, B.Sc. Thesis, Massachusets Institute of Technology (1939).

${ }^{33}$ P. C. Martin and J. Schwinger, Phys. Rev. 115, 1342 (1959).

${ }^{34}$ R. Puff and N. Gillis, Annals of Physics 46, 364 (1968).

${ }^{35}$ A. Savin and H.-J. Flad, International Journal of Quantum Chemistry 56, 327 (1995).

${ }^{36}$ T. Leininger, H. Stoll, H.-J. Werner, and A. Savin, Chem. Phys. Lett. 275, 151 (1997).

${ }^{37}$ I. C. Gerber and J. G. Àngyàn, Chem. Phys. Lett. 415, 100 (2005). 